\title{
Les alliances lydo-égyptienne et lydo-babylonienne
}

\author{
Kevin LELOUX*
}

\section{Introduction}

D'après Hérodote ${ }^{1}$, c'est au retour de la bataille indécise de Ptérie en Cappadoce lors de laquelle il s'est heurté à Cyrus, que Crésus décida de convoquer ses alliés pour attaquer à nouveau les Perses au printemps. Cette mention fournit l'occasion à l'historien d'Halicarnasse de dévoiler des alliances conclues auparavant par le roi de Lydie non seulement avec les Spartiates, mais aussi avec Amasis d'Égypte et même le roi de Babylone.

Dans ces quelques pages, nous reviendrons sur ces alliances lydo-égyptienne et lydo-babylonienne et explorerons les différentes questions qu'elles soulèvent à commencer par la crédibilité qu'il convient de leur reconnaître. Nous analyserons les deux alliances séparément, d'une part celle conclue entre Crésus et Amasis d'Égypte et d'autre part celle entre le roi lydien et le roi de Babylone. Nous étudierons par la même occasion les relations diplomatiques établies par Amasis avec les Cyrénéens et Polycrate de Samos ainsi que les rapports qui unissaient la Lydie à l'Égypte. De même, nous nous attarderons sur les liens diplomatiques tissés par le roi de Babylone, afin de mieux dégager quelle était la nature des rapports unissant ce dernier à Crésus de Lydie.

Pour ce faire, il est utile de rappeler le passage de l'historien d'Halicarnasse (I, 77), puisqu'il fera l'objet d'une analyse minutieuse :

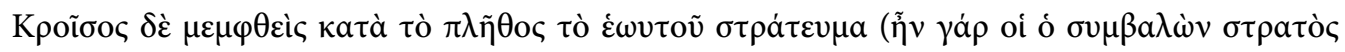

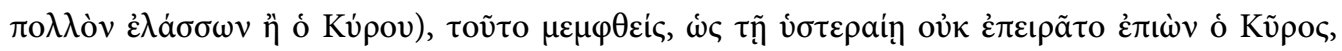

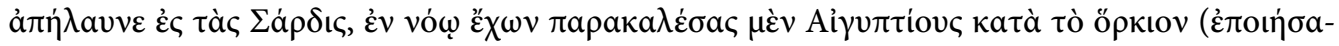

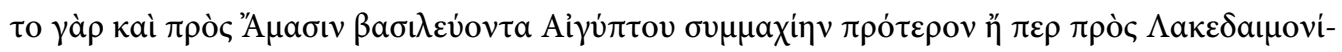

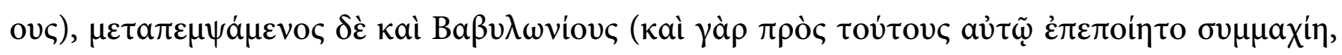

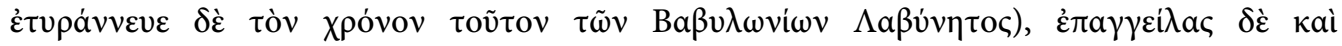

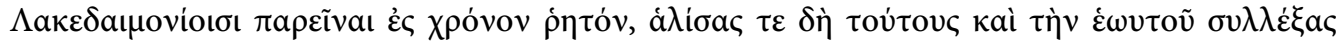

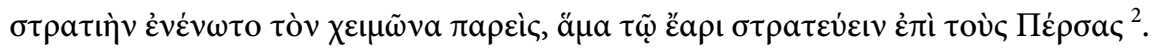

\footnotetext{
* Dr. Kevin Leloux, Université de Liège, Belgique (kevin.leloux@uliege.be ; (ㄱ https://orcid.org/00000002-5598-1446).

${ }^{1}$ Hérodote, I, 77.

${ }^{2}$ «Mais Crésus, mécontent de l'insuffisance numérique de ses troupes, - car les forces qui s'étaient engagées de son côté étaient bien moins nombreuses que celles de Cyrus, - mécontent de cette disproportion, comme le lendemain Cyrus ne tentait pas d'attaque, s'en retourna à Sardes. Son intention était d'appeler au secours les Égyptiens en vertu du traité (car il avait aussi conclu une alliance avec Amasis, roi d'Égypte, avant d'en conclure avec les Lacédémoniens), de faire venir également les Babyloniens (avec eux de même il avait une alliance ; le roi de Babylone était en ce temps Labynète), de mander en outre aux Lacédémoniens qu'ils eussent à se trouver à Sardes pour une date fixée ; ces alliés réunis, ses propres troupes rassemblées, il avait l'intention, après avoir laissé passer l'hiver, de marcher au printemps contre les Perses». Hérodote, I, 77. (éd. P. E. Legrand, Paris, Les Belles Lettres, 1932).
} 
Il faut remarquer que les alliances avec Amasis et le roi de Babylone sont explicitées dans deux incises. Dans la première, on trouve des précisions sur le traité en vertu duquel Crésus appelle

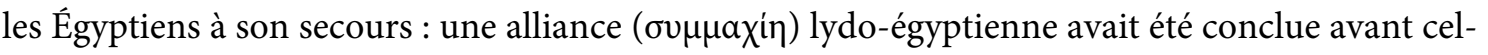
le avec Sparte. L'autre incise fournit la raison pour laquelle Crésus peut faire appel aux Babyloniens : une alliance ( $\sigma \nu \mu \mu \alpha \chi i \eta)$ avait également été conclue avec le roi babylonien de l'époque, celui qu'il nomme Labynète ${ }^{3}$.

\section{L'alliance avec Amasis d'Égypte}

Hérodote est le seul auteur à nous rapporter qu'une alliance avait été conclue entre Crésus et Amasis (ca. 570-526 av. J.-C.). Par ailleurs, cette mention ne se trouve qu'une seule fois dans son Enquête. Xénophon, de son côté, sans parler explicitement d'alliance - ce qui aurait encouragé à n'y voir qu'un simple emprunt à Hérodote, nous rapporte avec force détails la participation de troupes égyptiennes à la bataille de Thymbrara où ces dernières auraient d'ailleurs joué un rôle notable 4 . Penchons-nous sur le texte de Xénophon et analysons les passages de la Cyropédie où il est fait mention des troupes égyptiennes.

L'auteur transmet tout d'abord la quantité chiffrée des troupes égyptiennes qui viennent renforcer l'armée de Crésus à Thymbrara :

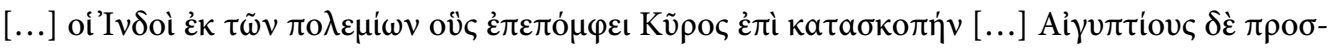

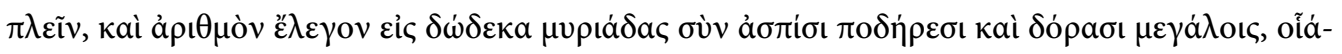

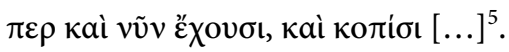

Une fois la bataille engagée et la défaite de Crésus toute proche, Xénophon relate la résistance des Égyptiens face aux troupes de Cyrus le Grand:

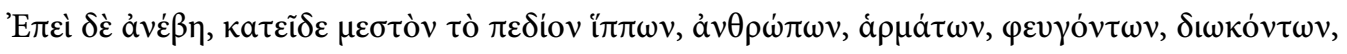

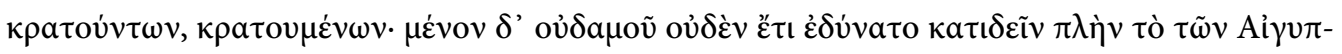

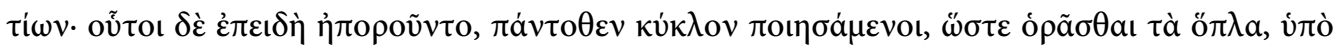

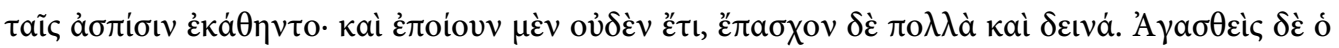

\footnotetext{
${ }^{3}$ Hérodote mentionne un certain Labynète. Il faut reconnaître ici Nabonide, roi de Babylone de 556 à 539 avant d'être détrôné par Cyrus. Hérodote mentionnait déjà au chapitre 74 un autre Labynète qui aurait servi de médiateur dans la conclusion de la paix entre la Lydie et les Mèdes en 585. Notre auteur précise au chapitre 188 toujours de son livre I que ce dernier Labynète était le père de celui qui avait conclu l'alliance avec Crésus. Or, il s'avère qu'en 585, le roi de Babylone était Nabuchodonosor et que ce dernier n'avait aucun lien de parenté avec Nabonide. Les propos d'Hérodote sont donc confus. Voir Asheri et al. 2007, 135 ; Legrand 1932a, 183, note 1.

${ }^{4}$ Xénophon, Cyropédie, VII, 1, 17-VII, 1, 45. Bataille opposant les troupes lydiennes aux unités perses devant la ville de Sardes $c a$. 547 av. J.-C. Celle-ci est relatée aussi chez Hérodote : I, 80 (qui toutefois ne relève pas à ce propos une participation égyptienne).

${ }^{5}$ «[...] les Indiens que Cyrus avait envoyés en mission d'espionnage, annonçant [...] que des Égyptiens arrivaient par mer, annonçant encore que leur nombre s'élevait à cent vingt mille hommes ( $\delta \dot{\omega} \delta \varepsilon \kappa \alpha$ $\mu v p ı d \dot{\delta} \delta \varsigma \varsigma$ ) armés de bouclier, de ces boucliers qui descendent jusqu'aux pieds, de ces longues lances qu'ils ont encore aujourd'hui ainsi que des cimeterres [...] ». Xénophon, Cyropédie, VI, 2, 9-10 (éd. M. Bizos, Paris, Les Belles Lettres, 1971 pour les livres I-II ; E. Delebecque, Paris, Les Belles Lettres, 1978 pour les livres VI-VIII).
} 


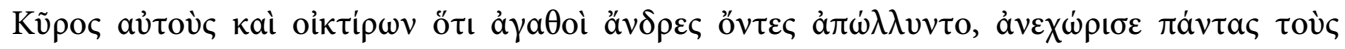

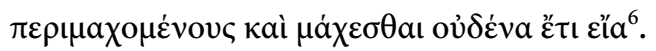

Il est vraisemblable que la mention des 12 myriades (120.000 hommes) d'Égyptiens présentes au sein de l'armée lydienne ne soit qu'un développement ultérieur de la part de Xénophon ou de sa source ${ }^{7}$. En effet, les précisions fournies sur les Égyptiens par l'Athénien pourraient surtout avoir été suscitées par le fruit de l'expérience géographique et militaire de l'auteur. ${ }^{8}$. Le mieux est sans doute de lier cette présence égyptienne évoquée dans son récit à la mort du roi de Sparte Agésilas (en 360 av. J.-C.) au retour de sa campagne d'Égypte (362-360 av. J.-C.). Xénophon avait servi sous ses ordres lors de sa campagne en Asie contre le satrape Tissapherne entre 396 et 394 av. J.-C. ${ }^{9}$. Il se serait ainsi renseigné sur cette dernière campagne du roi de Sparte et (en voulant rajouter aux propos de son célèbre prédécesseur) il aurait inséré dans sa Cyropédie ces informations recueillies sur les Égyptiens. C'est sans doute la raison pour laquelle Xénophon détaille l'ensemble des armes des soldats venus d'Égypte.

Bref, si notre auteur donne une place de choix aux Égyptiens autrefois à Thymbrara, c'est parce qu'il admire non pas tant l'Égypte du temps de Cyrus II, mais celle qui s'est rebellée récemment contre les Perses et pour laquelle Agésilas était parti se battre ${ }^{10}$. Ainsi, cet acharnement héroïque égyptien face aux troupes de Cyrus à Thymbrara pouvait sans doute s'imaginer au vu de la résistance des Égyptiens face aux Perses au début du IV siècle av. J.-C.

De plus, n'oublions pas que l'Athénien connaissait le récit d'Hérodote et plus particulièrement de son passage sur Crésus sur lequel il aurait cru adroit de rebondir ${ }^{11}$. En d'autres termes, c'est en cherchant à donner corps à l'évocation de l'alliance entre Crésus et Amasis chez Hérodote que Xénophon aurait inséré dans sa Cyropédie ce passage sur la présence et la résistance héroïque des Égyptiens à Thymbrara.

\footnotetext{
${ }^{6}$ «Une fois en haut, il [Cyrus] eut sous les yeux le spectacle d'une plaine couverte de chevaux, d'hommes, de chars, en train de fuir, de poursuivre, de vaincre, d'être vaincus ; mais nulle part il ne put voir un seul élément résister, sauf le corps des Égyptiens. Mais ceux-ci, lorsqu'ils furent aux abois, se tapirent, formés en un cercle parfait sous leurs boucliers de manière à laisser les armes seules visibles. Mais au lieu d'être un tant soit peu efficaces, ils subissaient une épreuve dure et terrible. Saisi d'admiration pour eux et déplorant que, guerriers héroïques, ils fussent en train de mourir, Cyrus ramena en arrière tous ceux qui combattaient autour du cercle et interdisait qu'on se battît davantage».Xénophon, Cyropédie, VII, 1, 40-41.

${ }^{7}$ Le nombre douze renvoie à l'idée de «totalité», de "groupe entier» : (cf. dodécapole ionienne, dodécapole éolienne, les douze principats d'Égypte (Hérodote, II, 147), les douze peuples fondateurs de l'amphictionie de Delphes, etc.). Sur la symbolique du nombre douze, voir Germain 1954, p. 54-56 et Fehling 1989, p. 231-232.

${ }^{8}$ Xénophon était parti en Asie, et durant cette campagne, il avait découvert des villes occupées par des Égyptiens. Notamment dans les Helléniques (III, 1, 7) où il mentionne la ville de Larisa appelée «l'égyptienne». Pour les deux villes qu'il mentionne dans la Cyropédie (VII, 1, 45), il a dû les voir lors de sa campagne en Asie. Delebecque 1957, p. 402. Sur la Cyropédie, voir aussi Tatum 1989.

${ }^{9}$ Anderson 2008, 146-162 ; Due 1989, 192-198 ; Canfora 1994, 379.

${ }^{10}$ Delebecque 1957, 403-404. Voir aussi Azoulay 2004, 202.

${ }^{11}$ Delebecque 1957, 393 ; Bizos 1971, V-VII ; Due 1989, 117-134.
} 
L'historien John Kinloch Anderson soutient aussi que la présence des troupes égyptiennes à la bataille de Thymbrara ne serait qu'une invention de la part de Xénophon ${ }^{12}$. Ainsi, lorsque Xénophon relate la reddition des Égyptiens à Cyrus et le don de villes fait par ce dernier en échange de leur fidélité après qu'ils ont déposé les armes, il nous signale que :

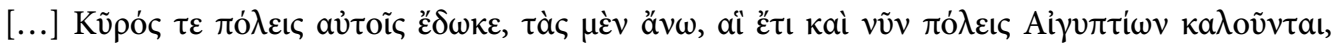

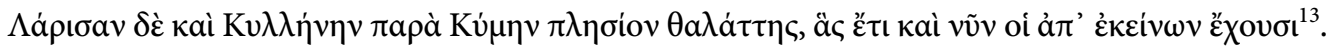

Cependant, le terme "égyptien» accordé à Larisa et Cyllène n'a pas encore lieu d'être du temps de Cyrus II puisque ce qualificatif représente l'état de l'empire perse au début du IV siècle av. J.C. Rien ne permet d'affirmer avec certitude que le don de ces villes par Cyrus aux anciens mercenaires égyptiens de Crésus fut effectué : les indications de Xénophon confirment simplement qu'il y avait de son temps des établissements égyptiens à Larisa et Cyllène ${ }^{14}$. En effet, du temps de Xénophon, des marins égyptiens étaient actifs sur les côtes occidentales d'Asie Mineure. Il n'est donc pas impossible que ces villes qualifiées d'égyptiennes aient été données par le roi de Perse ou l'un ou l'autre satrape à ces individus originaires d'Égypte ${ }^{15}$, mais bien après le conflit qui opposa Crésus à Cyrus.

Pour le reste, le récit de la bataille de Thymbrara que nous donne à lire Xénophon semble lui aussi imaginaire car comme invite à le penser J. K. Anderson, les stratégies mises en place par Cyrus et Crésus se présentent comme la décalque de celles des Spartiates et des Thébains ${ }^{16}$. Reste que la trame générale du récit de Xénophon s'inscrit sans fausse note dans le témoignage d'Hérodote. Maintenant que nous venons de laisser entrevoir que la présence de troupes égyptiennes dans l'armée de Crésus pourrait bien n'être qu'un développement imaginé par Xénophon afin de rendre son récit crédible, revenons au récit d'Hérodote.

L'historien d'Halicarnasse déclare qu'après avoir appris la chute de son beau-frère Astyage (le roi des Mèdes), Crésus aurait envoyé des ambassadeurs afin de tester la véracité de différents oracles et notamment celui du sanctuaire de Siwa en Libye ${ }^{17}$. Pour Vincenzo La Bua ${ }^{18}$, Crésus dépêcha justement ces ambassadeurs au sanctuaire en vertu de l'alliance conclue avec Amasis qui aurait joué un rôle dans les négociations entre Sparte et Crésus (en témoignerait l'envoi aux

12 Anderson 1970, 165-170.

${ }^{13}$ «Cyrus leur donna des villes, les unes dans l'intérieur, qui aujourd'hui encore sont appelées les 'villes égyptiennes', et, d'autre part, Larisa et Cyllène près de Cymé, non loin de la mer, que leurs descendants occupent encore aujourd'hui». Xénophon, Cyropédie, VII, 1, 45.

${ }^{14}$ Sekunda 1985, 19. Il ajoute que plus tard, on retrouve des noms d'Égyptiens dans ces régions. C'est le cas pour Tachos qui fonda Leukè entre Cymé et Clazomènes au IV ${ }^{\mathrm{e}}$ siècle av. J.-C. Il précise également que pour les établissements égyptiens situés à l'est de l'Halys, notre connaissance de cette région avant la conquête romaine est pauvre.

${ }^{15}$ Notamment à un certain Tamos, lieutenant-gouverneur d'Ionie sous le satrape Tissapherne (Thucydide, VIII, 87, 1-3). Anderson 1970, 168-169.

${ }^{16}$ Anderson 1970, 170-191. Sans compter que les Egyptiens sont présentés par Xénophon dans sa Cyropédie comme de «bons mercenaires», par opposition aux Chaldéens. Azoulay 2004, 216.

${ }^{17}$ Hérodote, I, 46.

${ }^{18}$ La Bua 1977, 39. 
Spartiates par Amasis d'une cuirasse que les Samiens auraient subtilisée lors du trajet) ${ }^{19}$. Ici, V. La Bua considère le système des alliances initié par Crésus comme une coalition quadripartite (Lydie - Sparte - Égypte - Babylone). Or, Hérodote évoque trois alliances bilatérales distinctes : une alliance entre Crésus et Lacédémone, une autre entre Crésus et Amasis et enfin une troisième entre Crésus et Labynète ${ }^{20}$.

Remarquons ensuite que V. La Bua tire des conclusions plutôt hâtives des maigres sources mises à sa disposition. De fait, il est vrai que pour Roland Crahay Crésus n'aurait pu envoyer des ambassadeurs à Siwa pour la simple raison qu'il ne pouvait connaître l'existence de ce sanctuaire. Pour R. Crahay, la liste des sanctuaires fournie par Hérodote n'aurait de sens que lorsque ces sanctuaires ne se seraient présentés comme des rivaux de Delphes qu'au V $\mathrm{V}^{\mathrm{e}}$ et non dès le VI $\mathrm{I}^{\mathrm{e}}$ siècle av. J.-C. ${ }^{21}$. Le sanctuaire de Siwa ayant été fondé seulement sous le règne d'Amasis (entre ca. 570 et 526 av. J.C. $)^{22}$, nous ne pouvons imaginer que Crésus ait pu avoir connaissance de l'existence d'un sanctuaire si lointain moins de vingt ans après sa création. Comment comprendre que ce sanctuaire libyen ait déjà acquis une renommée telle qu'il fût déjà célèbre en Lydie?

Dès lors, les arguments avancés par V. La Bua, et repris par certains chercheurs ${ }^{23}$, semblent perdre beaucoup de leur valeur puisque celui-ci s'appuie essentiellement sur la prétendue consultation par Crésus de l'oracle de Siwa ${ }^{24}$. De plus, V. La Bua fait valoir le récit de Xénophon évoquant la présence des troupes égyptiennes dans l'armée de Crésus à Thymbrara, épisode dont nous avons aussi relevé la nature probablement imaginaire. Pour en revenir aux sources disponibles du côté égyptien, il ne reste malheureusement pratiquement aucun vestige des stèles et monuments érigés par Amasis. L'explication avancée tiendrait au fait qu'à son arrivée en Égypte, Cambyse décida de faire marteler son nom et de persécuter la mémoire du pharaon, en témoignent notamment les sarcophages mutilés d'un fils et d'une des épouses d'Amasis ${ }^{25}$. Après avoir fait le point sur les sources disponibles et avoir convenu que le texte de Xénophon n'était peutêtre qu'une fiction de la part de l'auteur, penchons-nous maintenant sur la nature des liens qui auraient uni le dernier Mermnade au pharaon de la dynastie saïte.

\section{La nature de l'alliance conclue entre Crésus et Amasis}

Revenons au texte d'Hérodote et rappelons la phrase en I, 77 citée plus haut : «car il avait aussi conclu une alliance avec Amasis, roi d'Égypte, avant d'en conclure une avec les Lacédémoniens».

Le père de l'Histoire emploie le terme $\sigma v \mu \mu a x i^{26}{ }^{26}$ pour désigner le type de relation qui aurait uni

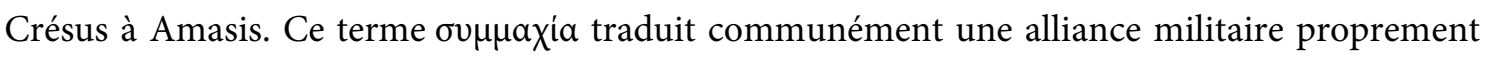
dite. Il désigne aussi bien le corps de troupes envoyé à la rescousse d'un allié, que la nature de la

\footnotetext{
${ }^{19}$ Hérodote, III, 47.

${ }^{20}$ Hérodote, I, 77.

${ }^{21}$ Crahay 1956, 195.

22 C'est Amasis qui prit la décision d'installer le siège d'un oracle à cet endroit. Voir Colin 1998, 329-354.

${ }^{23}$ Briant 1996, 910 et Asheri et al. 2007, 131-137 par exemple.

${ }^{24} \mathrm{La}$ Bua s'appuie aussi sur la véracité de l'existence de l'alliance entre Sparte et le roi lydien, une alliance étudiée dans Leloux 2014, 271-288.

${ }^{25}$ De Meulenaere 1975, col. 182 ; Bolshakov 2010, 45-53.

${ }^{26}$ Forme ionienne de $\sigma v \mu \mu a x i a$.
} 
relation qui existe entre deux ou plusieurs États à la suite d'un accord ${ }^{27}$. À l'époque d'Hérodote, $\sigma u \mu \mu \alpha \chi i$ renvoie tant à une alliance à finalité offensive que défensive ${ }^{28}$. La réalité qu'il recouvre

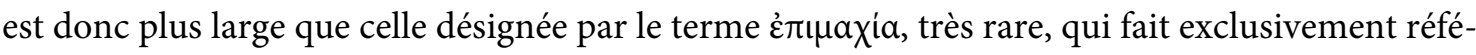
rence à une alliance de nature défensive ${ }^{29}$. Cette distinction terminologique ne sera attestée qu'à partir de la deuxième moitié du $\mathrm{V}^{\mathrm{e}}$ siècle ${ }^{30}$. Sans doute faut-il en déduire qu'avant l'émergence de cette restriction dans l'intensité de l'engagement convenu et encore à la période à laquelle écrit Hérodote, les obligations mutuelles contractées à la suite d'une alliance ( $\sigma \nu \mu \mu \alpha \chi i \alpha)$ comprenaient à la fois un volet offensif et un autre défensif ${ }^{31}$. Cependant, si dans la plupart des cas une

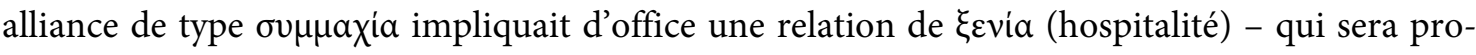

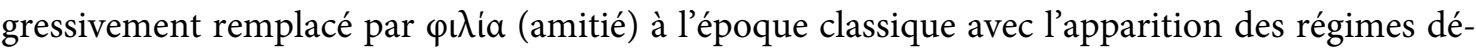
mocratiques ${ }^{32}$, en revanche l'affirmation de liens de $\xi \varepsilon v i ́ a$ n'impliquait pas ipso facto des liens de $\sigma v \mu \mu \alpha i^{33}$.

Ainsi, à lire Hérodote, Crésus aurait conclu une alliance à la fois de type défensif et offensif et aurait probablement au minimum entretenu des relations d'hospitalité avec le pharaon Amasis, bien qu'Hérodote ne l'évoque pas explicitement ${ }^{34}$. Pour nous aider à mieux définir quels liens avaient pu unir le roi de Lydie à Amasis, il convient d'examiner les relations qu'entretenait le pharaon avec les Cyrénéens d'une part et Polycrate de Samos d'autre part ${ }^{35}$.

\section{Les relations diplomatiques d'Amasis avec les Cyrénéens et Polycrate de Samos :}

Toujours selon Hérodote, Crésus ne serait pas le seul avec qui Amasis aurait lié son sort. En effet, selon lui, le pharaon aurait conclu amitié et alliance avec les Cyrénéens (Kvpqvaioı $\delta \grave{\varepsilon}$

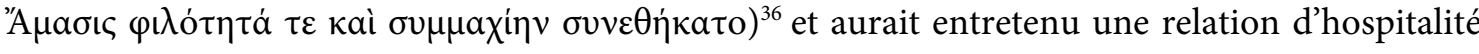

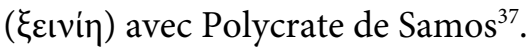

${ }^{27}$ Giovannini 2007, 229 ; Kaplan 2016, 143.

${ }^{28}$ Sur la $\sigma \nu \mu \mu \alpha x i \alpha$, voir Couvenhes 2016.

${ }^{29}$ Giovannini 2007, 241-244 ; Couvenhes 2016, 27-30. Le terme se rencontre pour la première fois chez Thucydide qui mentionne une alliance défensive entre Athènes et Corcyre (Thucydide, I, 44). Sur les rares

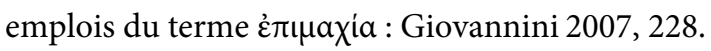

${ }^{30}$ Bederman 2001, 162 et Giovannini 2007, 228-230.

${ }^{31}$ Bederman 2001, 162 ; Adcock - Mosley 1974, 122 ; Couvenhes 2016, 29-30 ; Kaplan 2016, 143.

${ }^{32}$ Giovannini 2007, 228 ; Intrieri 2010 ; 2013, 130-133.

${ }^{33}$ Adcock - Mosley 1974, 206-209; voir aussi Kaplan 2016, 137-140.

${ }^{34} \mathrm{Au}$ contraire de ce qu'il fait en I, 69-70 lorsqu'il évoque l'alliance entre Crésus et Lacédémone. Cependant, dans ce cas précis, Hérodote rentre beaucoup plus dans les détails entourant la conclusion de cette $\sigma u \mu \mu \alpha x i \alpha$. Les liens de $\xi \varepsilon v i \alpha$ impliquent que les deux parties ne sont pas parentes, mais leurs relations sont semblables à celles de parents. Sur la Ǧvía en général : Herman 1987 et son compte rendu par Schmitt-Pantel 1990, 878-879. Voir aussi la note 14 de Kaplan 2016, 137 pour une bibliographie récente.

${ }^{35}$ Sur l'alliance entre Amasis et Polycrate, voir l'article récent de Philip Kaplan. Kaplan 2016, 132-157.

${ }^{36}$ Hérodote, II, 181.

${ }^{37}$ «Il fit des offrandes à Samos à cause des relations d'hospitalité qui existaient entre lui et Polycrate fils d'Aiakes». Hérodote, II, 182. Nous avons ici un bel exemple du type de cadeaux que se faisaient deux États «amis». 
Ces rapports privilégiés qu'il aurait entretenus avec Cyrène nous font penser davantage à une relation que les Grecs qualifient de $\xi \varepsilon v i a$ qu'à une alliance militaire au sens étroit du terme. En tout cas, Hérodote nous apprend qu'Amasis aurait envoyé à Cyrène une statue dorée d'Athéna. En retour, le pharaon aurait reçu comme épouse une nommée Ladiké, probablement une fille de Battos (roi de Cyrène) ${ }^{38}$. Cette relation d'hospitalité liant désormais le pharaon au roi de Cyrène était sans doute née des conditions particulières qui avaient entouré l'accession au trône d'Amasis : pour se faire proclamer pharaon, ce dernier avait profité d'une révolte de l'armée égyptienne lors d'une expédition menée par Apriès contre Cyrène ${ }^{39}$. Une bataille entre les troupes formées d'Égyptiens sous le commandement d'Amasis et les mercenaires grecs qui servaient dans l'armée égyptienne sous le commandement d'Apriès s'était ensuivie. Ce dernier vaincu, Amasis s'était ainsi imposé comme pharaon non sans violence ${ }^{40}$.

Installé sur le trône au terme de cette passe d'armes, Amasis aurait donc décidé de regagner les faveurs des Grecs (en particulier des gens de Cyrène mais aussi les mercenaires servant dans l'armée égyptienne) en leur offrant des présents, en se liant d'amitié avec certains d'entre eux, ainsi qu'en leur fournissant des privilèges à Naucratis ${ }^{41}$. Il est fort probable néanmoins que cette relation, qui était perçue aux yeux des Grecs comme étant des liens d'hospitalité, était plutôt considérée par le pharaon comme étant une alliance conclue entre partenaires inégaux : un souverain (Amasis) et un vassal (les Cyrénéens) ${ }^{42}$. Cependant, vu l'absence de tablettes ou de textes officiels retrouvés en Égypte (et même en Lydie), et contemporains de la période qui nous préoccupe, il est difficile de cerner avec exactitude les détails de telles relations.

Pour Amasis, donner un cadre institutionnel à la présence de Grecs à Naucratis (déjà présents dès le début $\mathrm{du} \mathrm{VI}{ }^{\mathrm{e}}$ siècle av. J.-C.) $)^{43}$ se révélait bénéfique : l'établissement grec était situé sur la branche canopique du Nil, juste à une dizaine de kilomètres de Saïs. Comme sa capitale lui servait de port, cet emporion était source de revenus pour le pharaon, une taxe étant prélevée sur toutes les importations qui arrivaient à Naucratis et sur les biens produits à cet endroit. L'autre intérêt de ce rapprochement avec les Grecs fréquentant la Méditerranée orientale est qu’il lui aurait permis d'endiguer la «colonisation intérieure» du territoire égyptien, une pénétration opérée jusque-là de façon anarchique par ces Grecs ${ }^{44}$.

Sans doute est-ce également pour les avantages qu'il entendait bien tirer de la prospérité montante de Naucratis qu'Amasis décida d'envoyer des offrandes au sanctuaire d'Athéna de Lindos à Rhodes dont les commerçants n'étaient pas les derniers à fréquenter l'emporion où ils jouaient un rôle actif dans l'administration de l'Hellénion ${ }^{45}$. Cela dit, pour Hérodote, la raison de son

${ }^{38}$ Hérodote, II, 181.

${ }^{39}$ Voir Jansen-Winkeln 2014 ; Kaplan 2016, 148.

${ }^{40}$ Hérodote, II, 161-172.

${ }^{41}$ Hérodote, II, 178-179.

${ }^{42}$ Kaplan 2016, 148. Sur les traités inégalitaires dans le Proche-Orient ancien, voir Charpin 2019, 135-137.

${ }^{43}$ Les céramiques grecques les plus anciennes retrouvées à Naucratis datent du début du $\mathrm{VI}^{\mathrm{e}}$ siècle av. J.-C. Agut-Labordère 2012b, 360.

${ }^{44}$ Braun 1982, 37-43 ; Baurain 1997, 304 ; Greaves 2010, 167 ; Agut-Labordère, 2012b, 353-373 ; Redon 2012, 55-93.

${ }^{45}$ Bresson 1980, 308-310. Sur l'Hellénion, voir aussi Bresson 2005, 139-152. 
geste était la suivante : ce n'est pas à cause de relations d'hospitalité mais parce que «le temple d'Athéna à Lindos a été fondé, à ce qu'on dit, par les filles de Danaos, qui abordèrent là en fuyant les fils d'Égyptos» ${ }^{46}$. En réalité, les motivations devaient être à la fois de nature politique et économique : Amasis entendait établir de bonnes relations avec $\operatorname{Lindos}{ }^{47}$, ceci afin d'encourager, notamment, le commerce des Grecs avec l'emporion de Naucratis ${ }^{48}$. Hérodote évoque par ailleurs bien le fait que la cité de Lindos était présente à Naucratis et était une des cités en charge de la gestion de son sanctuaire commun : l'Hellénion ${ }^{49}$.

Les liens tissés par Amasis avec Polycrate de Samos auraient également résulté, entre autres, de motifs économiques liés à Naucratis ${ }^{50}$. En effet, Hérodote y mentionne aussi la présence des Samiens, où ils avaient fondé et administraient un sanctuaire d'Héra ${ }^{51}$. Selon l'historien d'Halicarnasse, Polycrate «avait conclu un traité d'amitié avec Amasis roi d'Égypte, à qui il envoyait des

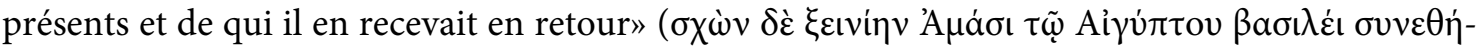

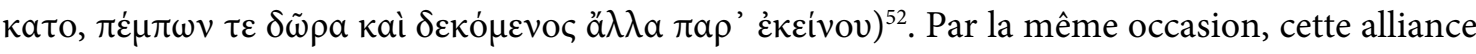
aurait aussi permis à l'Égypte, grâce à la flotte samienne, de se prémunir d'une attaque navale des Perses qui venaient de renverser le royaume de Babylone (en 539 av. J.-C.) et de les contenir le long du littoral anatolien ${ }^{53}$. Ici aussi, ces liens auraient été perçus de manière différente par Amasis et par Polycrate. Le tyran samien aurait vu cette relation comme un traité d'hospitalité entre partenaires égaux, tandis que le pharaon aurait considéré cette alliance davantage comme un traité dissymétrique entre un «vassal» et son seigneur. Du point de vue égyptien, Samos était considéré comme une puissance mineure dirigée par un «escroc»" ${ }^{54}$. Mais ce traité aurait été dénoncé plus tard par Amasis : ce dernier craignait, si la fortune de Polycrate venait à tourner et

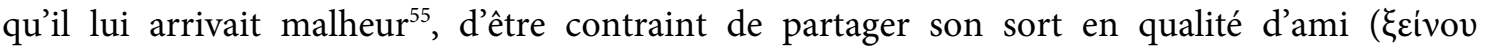

${ }^{46}$ Il dédia à l'Athéna de Lindos deux statues en pierre et une cuirasse de lin brodée de coton et d'or. Hérodote, II, 182 et III, 47.

${ }^{47}$ Les pharaons passant pour les descendants d'Égyptos, Amasis entendait ainsi réparer l'outrage perpétré par Égyptos envers les filles de Danaos et faire table rase des contentieux du passé.

${ }^{48}$ Au début du règne d'Amasis, Lindos était entre les mains du tyran Cléobule, un des Sept Sages. Voir Francis - Vickers 1984, 68-69.

${ }^{49}$ Hérodote, II, 178. L'Hellénion était administré par neuf cités : quatre ioniennes (Chios, Téos, Phocée et Clazomènes), quatre doriennes (Rhodes, Cnide, Halicarnasse et Phasélis) et une éolienne (Mytilène). Pour Rhodes, les trois cités de l'île (Camiros, Ialysos et Lindos) agissaient de concert pour l'administration du sanctuaire. Bresson 1980, 308 ; 2005, 139-142.

${ }^{50}$ Sur l'alliance entre Amasis et Polycrate, voir Kaplan 2016.

${ }^{51}$ Hérodote, II, 178.

${ }^{52}$ Hérodote, III, 39.

${ }^{53}$ Kaplan 2016, 149.

${ }^{54}$ Kaplan 2016, 150, à la manière des traités néo-assyriens. Voir Charpin 2019, 245-255.

${ }^{55}$ Hérodote, III, 39-43. 
$\grave{a} v \delta \rho o ́ \varsigma)$. En réalité, si Amasis rompit l'alliance, c'est plus probablement en raison d'un manque de réciprocité de la part du tyran samien ${ }^{56}$.

Ce pharaon décidément en quête de l'amitié des Grecs est aussi crédité du financement d'une bonne part de la reconstruction du temple d'Apollon de Delphes rendue nécessaire après un incendie fortuit daté de 548 av. J.-C. sur base du témoignage de Pausanias ${ }^{57}$.

Certains historiens (comme V. La Bua ${ }^{58}$ vont même jusqu'à estimer qu'Amasis, comme Crésus, avait conclu une alliance avec la cité de Sparte : en témoignerait la cuirasse qu'il aurait offerte aux Spartiates mais que les Samiens auraient dérobée un an avant le «vol du cratère» ${ }^{59}$, celui envoyé par Lacédémone à Sardes en reconnaissance de leur alliance convenue avec Crésus ${ }^{60}$. Cette mention d'un tel cadeau offert par Amasis à Sparte n'est peut-être qu'un ajout par Hérodote d'une information qu'il aurait glanée à Delphes de sources favorables à la cité laconienne : on sait en effet qu'Hérodote doit avoir effectué son voyage en Grèce continentale avant son départ pour Thourioi, aux alentours des années $447-444 / 3$ av. J.-C. ${ }^{61}$, et que, d'autre part, Sparte a exercé une forte influence sur le sanctuaire phocidien tout au long du $\mathrm{V}^{\mathrm{e}}$ siècle, en particulier après la bataille de Coronée (447 av. J.-C.) ${ }^{62}$.

Les vols du cratère destiné à Crésus et de la cuirasse d'Amasis ont fourni un motif aux Spartiates pour prêter main-forte aux Samiens contre Polycrate. Cependant, ces derniers présentaient une tout autre version, prétendant que par leur aide, les Lacédémoniens «s'acquittaient d'une dette de reconnaissance, parce que les Samiens antérieurement les avaient assistés avec des navires contre les Messéniens» ${ }^{63}$. Or, lorsqu'il mentionne les versions divergentes des Samiens et des Spartiates au sujet du «vol» du cratère, par sa tournure de phrase Hérodote invitait déjà ses auditeurs à préférer la version des gens de Samos et à discréditer celle de Lacédémone ${ }^{64}$.

Peut-être faudrait-il, à l'instar de la version samienne du «vol du cratère», privilégier la version des Samiens dans le cas de l'aide apportée par Sparte contre Polycrate. Si la cuirasse d'Amasis offerte à Lindos semble avoir réellement existé (pourrait en témoigner le catalogue des offrandes

\footnotetext{
${ }^{56}$ Kaplan 2016, 151. Les navires samiens devaient aussi s'adonner à des actes de piraterie à l'encontre des navires grecs entre l'Égée et l'Égypte, mettant à mal la politique du pharaon de recherche d'amitiés dans le monde grec.

${ }^{57}$ Hérodote, II, 180 ; Pausanias, X, 5, 13.

${ }^{58}$ La Bua 1977, 40-43.

${ }^{59}$ Hérodote, I, 70.

${ }^{60}$ Hérodote, III, 47. Sur la datation de ces vols, voir infra.

${ }^{61}$ Voir l'ouvrage introductif de Legrand dans la collection Budé : Legrand 1932b, 29-32 et Lenfant $2011,215$.

${ }^{62}$ Sordi 1957, p. 64. Sur l'influence de Sparte sur le sanctuaire de Delphes : Daux 1957, 114-116 ; Malkin 1989, 129-153 et Sánchez 2001, 114.

${ }^{63}$ Hérodote, III, 47.

${ }^{64}$ Voir le commentaire du chapitre 70 du livre I d'Hérodote : Legrand 1932a, 74, note 2.
} 
du sanctuaire réalisé au $\mathrm{I}^{\mathrm{er}}$ siècle av. J.-C. ${ }^{65}$, nous pouvons cependant mettre en doute l'existence de celle destinée à Lacédémone.

De plus, même si nous sommes incapables de dater le vol de la cuirasse ainsi que celui du cratère ${ }^{66}$, il parait plus probable de convenir que Polycrate «qui rendait à ses amis ce qu'il leur avait volé» ${ }^{67}$ ait retourné cette cuirasse à Amasis avec lequel il entretenait des liens qu'il considérait

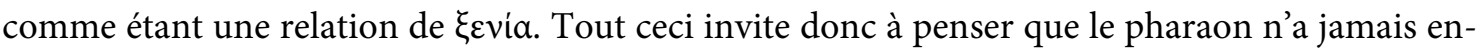
voyé de cuirasse en cadeau à Sparte. Après nous être intéressé sur les relations entre Amasis et les Cyrénéens et entre ce même pharaon et Polycrate de Samos, venons-en maintenant aux liens entre les Mermnades et l'Égypte.

\section{Les relations entre l'Égypte et la Lydie}

Pour ce qui est de la Lydie, rappelons que par le passé, Gygès s'était déjà lié à l'Égypte, en particulier avec le pharaon Psammétique pour lequel le roi de Lydie avait envoyé des mercenaires cariens et ioniens ${ }^{68}$. Ces «hommes de bronze venus de la mer», comme les décrit Hérodote ${ }^{69}$, auraient servi lors de la campagne de réunification de l'Égypte par Psammétique contre son rival napatéen Sabacos (Shabaka) ${ }^{70}$.

Si des liens ont uni Crésus au pharaon Amasis, ceux-ci ont dû se conformer aux usages en vigueur dans le monde oriental ${ }^{71}$. Les Grecs les auraient assimilés à des liens de $\xi_{\varepsilon v i ́ a}$. En effet, nous venons de le voir, les différentes relations qu'entretenait Amasis avec ses «alliés» grecs reposaient sur des liens dissymétriques liant un seigneur et son «vassal», perçus aux yeux des Grecs comme étant des accords noués entre individus égaux. Ce qui n'était manifestement pas le cas dans le chef du pharaon qui considérait ses «alliés» comme ses subordonnés.

Différentes hypothèses pourraient expliquer la recherche de la part de Crésus d'une alliance avec Amasis.

${ }^{65}$ Anagraphe von Lindos, FGrH 532 F 1 J, ligne 29. Des restes d'une statuette en basalte de provenance égyptienne datés du VI ${ }^{\mathrm{e}}$ siècle av. J.-C. ont été retrouvés lors des fouilles du sanctuaire. Francis - Vickers 1984, 69. Voir aussi Higbie 2003, 113-115 ; Dillery 2005, 516 ; Massart 2006, 235-236.

${ }^{66}$ Hérodote donne deux moments différents pour le vol du cratère : 1) juste avant la prise de Sardes par Cyrus ; 2) sous le règne d'Alyatte ( $c a .610-560$ av. J.-C.) et la tyrannie de Périandre de Corinthe (ca. 625-585 av. J.-C.). Le détournement de la cuirasse s'étant déroulé un an avant celui du cratère, le larcin aurait donc été réalisé soit en 548 soit entre 610 et 585 av. J.-C. (dates communes à Périandre et Alyatte). Mais Amasis étant monté sur le trône en 570 av. J.-C., la dernière possibilité peut donc être écartée, ainsi, le vol de la cuirasse se serait donc déroulé en 548 av. J.-C. (également l'année de l'incendie du grand temple de Delphes).

${ }^{67}$ Hérodote, III, 39.

${ }^{68}$ Cette mention de l'envoi d'aide de la part de Gygès (Guggu) se trouve par ailleurs dans les sources assyriennes. Pour l'édition et la traduction des sources assyriennes, voir l'ouvrage récent de Novotny Jeffers 2018 (RINAP 5/1). Le passage mentionné ici correspond à RINAP 5/1 11 col. ii 1l. 114-115.

${ }^{69}$ Hérodote, II, 152. Pour les mercenaires grecs en Égypte, voir Agut-Labordère 2012a, 293-306.

${ }^{70}$ Spalinger 1978, 400-409; James 1991, 711.

${ }^{71}$ Sur les alliances au Proche-Orient, voir ouvrage récent de Charpin 2019. 
La première voudrait que Crésus, avant la chute d'Ecbatane $c a .550$ av. J.-C. et après ses campagnes contre les différentes cités grecques ${ }^{72}$, ait cherché à conclure une alliance avec le pharaon afin de faciliter le commerce des Grecs à Naucratis. Cette alliance, probablement conclue à dis$\operatorname{tance}^{73}$, devait sans doute comporter des clauses politiques (ne pas être hostile envers l'autre partie) et militaires (s'engager à avoir les mêmes amis et ennemis et à envoyer de l'aide en cas de conflits par exemple ${ }^{74}$ accompagnées de clauses commerciales (telles que celles conclues auparavant par les Assyriens dans le cadre de leur commerce à longue distance avec leurs comptoirs commerciaux en Anatolie, mais aussi celles des traités hittites et néo-assyriens) ${ }^{75}$. Dans ce cas, Amasis aurait accepté cette alliance car, comme Lacédémone lors de la conclusion de l'alliance lydo-spartiate ${ }^{76}$, il n'imaginait sans doute pas devoir fournir une aide militaire à Crésus qui passait pour le souverain le plus puissant d'Anatolie. Ainsi, étant donné que le pharaon prélevait une taxe sur les échanges commerciaux à Naucratis, cette alliance devait-elle servir les intérêts des deux royaumes ${ }^{77}$. En effet, d'une part, ce rapprochement ne pouvait qu'influer favorablement sur le commerce de l'Égypte en Méditerranée orientale que le pharaon entendait favori$\operatorname{ser}^{78}$; et d'autre part, il rencontrait aussi à Naucratis les intérêts des Grecs sous tutelle lydienne, car le commerce était à côté de son très probable rôle de porte d'accès des mercenaires en Égypte la principale raison de leur présence récurrente en ces lieux ${ }^{79}$; Milet, en particulier, inféodée à l'État lydien, avait assurément de gros intérêts à défendre dans l'emporion de Naucratis ${ }^{80}$.

La deuxième hypothèse voudrait que Crésus ait cherché à nouer des liens avec Amasis après la chute d'Ecbatane ca. 550 av. J.-C. ${ }^{81}$. Dans ce cas-ci également, cette alliance aurait comporté des clauses politiques, militaires et commerciales (toujours pour faciliter le commerce à Naucratis). Cependant, ici, Crésus aurait davantage noué ces accords afin de mener à bien sa campagne de

${ }^{72}$ Hérodote $(\mathrm{I}, 26)$ déclare que Crésus entama son règne par des campagnes à l'encontre des cités grecques d'Asie Mineure.

${ }^{73}$ Puisqu'aucun texte ne mentionne un voyage de Crésus en Égypte ou d'Amasis en Lydie.

${ }^{74}$ Kaplan 2016, 141-142 ; Charpin 2019, 141-158.

${ }^{75}$ Charpin 2019, 163-167.

${ }^{76}$ Leloux 2014, 283-284.

${ }^{77}$ A. Spalinger affirme que la politique égyptienne au Levant, de la fin $\mathrm{VII}^{\mathrm{e}}$ jusqu'au premier quart $\mathrm{VI}^{\mathrm{e}}$ siècle av. J.-C., avait essentiellement un but commercial. Spalinger 1977, 222. Pour A. Fantalkin, Naucratis aurait été fondée par Milet à la suite du traité de paix conclu avec Alyatte. Les Mermnades ont pu alors tirer, eux aussi, à la suite de l'initiative milésienne, un bénéfice commercial de ce comptoir grec en Égypte. Fantalkin 2014, 17-52.

${ }^{78}$ Spalinger 1977, 222-223 ; Fantalkin 2014, 43-44. Selon Diodore de Sicile (I, 68) le prédécesseur d'Amasis, Apriès, avait vaincu les Phéniciens et Chypriotes au cours d'un combat naval. L'intérêt des pharaons pour Chypre s'inscrit dans cette volonté de dominer la Méditerranée orientale. Masson 1971, 28-46 ; Balandier 2009, 78-96.

${ }^{79}$ Cook 1982, 212.

${ }^{80}$ Milet avait besoin du grain qu'elle ne pouvait fournir seule pour subvenir aux besoins de sa population. Greaves 2002, 101-104; 2010, 70-76 et 131-132.

${ }^{81}$ Mais cela va à l'encontre de ce qu'avance Hérodote : Crésus aurait noué une alliance avec Amasis avant d'en conclure une avec Sparte après avoir appris la chute d'Ecbatane. Hérodote, I, 46-77. 
conquête en Anatolie et en Ptérie ${ }^{82}$, plutôt que de trouver un allié contre un ennemi commun aux Lydiens et aux Egyptiens puisque Cyrus ne devait pas encore être considéré par Amasis ca. 550 av. J.-C. comme étant une menace sérieuse pour ses possessions ${ }^{83}$. Crésus pensait ainsi pouvoir compter sur des forces supplémentaires pour attaquer la région à l'est de l'Halys. Toutefois, il existe une objection à cette hypothèse d'une alliance offensive : quel intérêt avait Amasis à s'engager militairement dans une région si lointaine alors qu'il redoutait à tout moment une attaque des troupes babyloniennes au nord ${ }^{84}$ ? On peut également s'interroger sur l'absence d'aide égyptienne envoyée par Amasis à Crésus lors du siège de la citadelle de Sardes par les troupes perses ${ }^{85}$.

D'une part, si le pharaon considérait cette alliance - à l'instar de celles qu'il avait nouées avec Polycrate et le roi de Cyrène - comme étant conclue avec un subalterne, Amasis n'avait aucune obligation d'envoyer des troupes armées prêter main forte à son «vassal» ${ }^{86}$. Néanmoins cette supposition est fort peu probable car Crésus devait être considéré par le pharaon égyptien comme son alter ego, son «frère». Après la soumission de l'ensemble des cités grecques de la frange égéenne de l'Asie mineure, Crésus devait être considéré par celles-ci comme étant le souverain le plus puissant du moment. Dès lors, par l'intermédiaire des Grecs fréquentant Naucratis, notamment, Amasis a pu s'informer de la puissance et du statut de ce souverain contrôlant presque l'ensemble de l'Anatolie. D'autre part, si l'alliance était conclue entre partenaires égaux, il se pourrait que, comme le sous-entend Hérodote ${ }^{87}$, le rassemblement des troupes égyptiennes, prévues pour prêter main forte au Mermnade, avant leur départ pour Sardes ait pris du retard et que la nouvelle de la chute de la capitale lydienne arriva à Saïs avant même le départ des contingents. Cette dernière explication signifie que les clauses de nature militaire de la prétendue alliance entre Amasis étaient résolument défensives ${ }^{88}$, et met ainsi à mal l'hypothèse selon laquelle le roi lydien aurait pu conclure son alliance après la chute d'Ecbatane ca. 550 av. J.-C. ${ }^{89}$.

Sur ces constatations, les deux souverains auraient ainsi noué des liens avant la chute du royaume mède et la prise de sa capitale par les Perses. Cette alliance devait dès lors comporter des clauses politiques, militaires probablement de nature exclusivement défensive (à en croire Hérodote), ainsi que des clauses commerciales. Cependant, pour quelles raisons ces deux rois au-

${ }^{82}$ Leloux 2018, 313-318.

${ }^{83}$ Cela changera après la chute de Babylone en 539 av. J.-C. Kaplan 2016, 149.

${ }^{84}$ Babylone contrôlait la Syrie-Palestine depuis la chute de Jérusalem en ca. 587 av. J.-C. et menaçait les possession égyptiennes aux alentours de Gaza au début du règne d'Amasis. Roux 1995, 425 ; Elayi 2018, 308-310.

${ }^{85}$ On ne peut totalement exclure l'envoi par Amasis de mercenaires grecs engagés en Égypte pour prêter main forte à Crésus, mais Hérodote en tout cas ne le mentionne pas. Il indique simplement la présence des mercenaires (grecs) de Crésus.

${ }^{86}$ Les engagements ne sont pas symétriques entre partenaires inégaux. Charpin 2019, 155-156.

${ }^{87}$ Hérodote, I, 77.

${ }^{88}$ Sur les alliances défensives au Proche-Orient ancien, voir Charpin 2019, 154.

${ }^{89}$ D’autant plus que comme le signale Hérodote, Crésus aurait conclu cette alliance avec Amasis avant celle qu'il aurait conclue avec Lacédémone. Elle même nouée après que Crésus a appris la chute d'Astyage (ca. 550 av. J.-C.). Hérodote, I, 46 et 77. 
raient-ils conclu une alliance défensive avant 550 av. J.-C. ? Peut-être faut-il se tourner du côté du royaume de Babylone.

À la fin $\mathrm{du} \mathrm{VII}^{\mathrm{e}}$ et au début du VI ${ }^{\mathrm{e}}$ siècle av. J.-C., les pharaons égyptiens durent faire face régulièrement aux armées babyloniennes en Syrie-Palestine, territoire sous contrôle égyptien depuis la victoire de Nékao II à Megiddo en 609 av. J.-C.. Nékao II, Psammétique II et Apriès tentèrent de contenir l'avancée des troupes babyloniennes mais la prise de Jérusalem en 587 par Nabuchodonosor II (ca. 605-562 av. J.-C.) marqua la fin de la présence égyptienne dans cette région ${ }^{90}$. En 568 av. J.-C., Amasis a dû lui aussi faire face aux armées du roi babylonien ${ }^{91}$, mais entre la mort de Nabuchonodosor II ca. 562 (Crésus montant sur le trône en ca. 561) et 556 av. J.-C., Amasis n'a pas dû redouter une attaque des rois babyloniens Amêl-Marduk et Neriglissar dont les campagnes militaires étaient plutôt axées vers le nord et la Cilicie. Cependant, avec l'arrivée au pouvoir de Nabonide et ses campagnes militaires à l'ouest, à proximité de Gaza et la frontière de l'Égypte ${ }^{92}$, Amasis a probablement craint, à nouveau, une attaque des armées babyloniennes sur ses possessions septentrionales. Ces activités militaires à proximité des territoires égyptiens pourraient expliquer une recherche de la part du pharaon d'un allié afin de contrer la puissance de Nabonide si elle venait à attaquer l'Égypte. Ainsi, Amasis aurait conclu une alliance défensive avec un autre souverain puissant du moment : Crésus. De son côté, le roi lydien aurait pu, lui aussi, chercher à se lier avec le pharaon afin de pouvoir compter sur des troupes supplémentaires en cas d'attaque de l'armée babylonienne présente en Cilicie depuis le règne de Nabuchodonosor et de ses successeurs AmêlMarduk et Neriglissar ${ }^{93}$. La présence de Babylone dans cette région frontalière avec les domaines sous contrôle lydien ${ }^{94}$, pouvait constituer, aux yeux du Mermnade, comme étant une menace pour ses propres possessions en cas d'attaque des Chaldéens.

Crésus et Amasis auraient ainsi pu conclure une alliance comportant des clauses politiques : avoir les mêmes amis et ennemis (l'ennemi commun étant le royaume de Babylone); des clauses militaires : se prêter assistance en cas d'attaque d'un tiers (alliance défensive) ; des clauses commerciales : favoriser le commerce des Grecs à Naucratis ${ }^{95}$. Dans ce cas-ci, l'absence d'aide envoyée par Amasis pourrait s'expliquer par le fait que l'alliance devait comporter une clause de fidélité politique : s'engager à ne pas conclure de paix séparée ni même des relations diplomatiques avec l'ennemi ${ }^{96}$. Comme nous l'analyserons ci-après, Crésus ayant conclu (ultérieurement à l'alliance avec Amasis) un accord avec Nabonide de Babylone, le pharaon n'était plus tenu de respecter ses engagements puisqu'une clause de l'alliance avec Crésus n'avait pas été respectée.

Reste que, dans l'état actuel de la documentation, si une quelconque relation (qu'Hérodote qua-

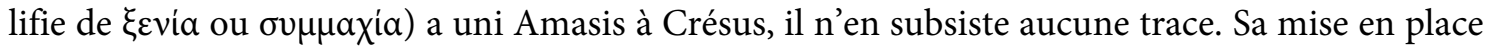
éventuelle - avec l'envoi de plusieurs ambassades à Saïs et sa mise par écrit sur tablettes - a pu

\footnotetext{
${ }^{90}$ Roux 1995, 423-425 ; Briant 1996, 33, 54-58 ; Joannès 2008, 209-216 ; Winand 2017, 315-350 ; Elayi 2018, 283-310.

${ }^{91}$ Spalinger 1977, 238-244 ; Roux 1995, 425 ; Elayi 2018, 308-310.

${ }^{92}$ Elayi 2018, 311-322.

${ }^{93}$ Hawkins 1982, 434 ; Wiseman 1991, 235 ; Joannès 1991, 262-266 ; Roux 1995, 426.

${ }^{94}$ Hérodote, I, 28.

${ }^{95}$ Charpin 2019, 163-167.

${ }^{96}$ Sur les clauses de fidélité politique, voir Charpin 2019, 142-154.
} 
s'accompagner d'échanges de présents, voire d'un mariage inter-dynastique, à la mode procheorientale pluriséculaire $^{97}$. Mais ni l'historien grec ni aucune autre source grecque ou orientale n'y font allusion ${ }^{98}$.

Sur ces constatations, une dernière hypothèse reste à envisager : Crésus et Amasis ne conclurent jamais une quelconque alliance comme nous le transmet le seul Hérodote. Peut-être ce lien fort entre Crésus et le pharaon Amasis a-t-il été imaginé dès le début du V viècle av. J.-C. à Delphes où ces deux souverains - personnages exceptionnels aux yeux des Grecs à peine sortis des guerres médiques - s'étaient montrés très généreux (en témoignaient la donation d'Amasis de 1000 talents d'alun pour la coûteuse reconstruction du «quatrième» temple après l'incendie de 548 av. J.-C. et les nombreuses offrandes antérieures de Crésus) : ces deux monarques s'étaient imposés dans l'imaginaire grec comme ceux qui avaient été naguère à la tête des deux royaumes les plus puissants de cette époque et qu'avaient balayés les Perses eux-mêmes bientôt bousculés par les Grecs dans les guerres médiques.

Venons-en maintenant au second volet de notre analyse, l'alliance lydo-babylonnienne.

\section{L'alliance de Crésus et Nabonide de Babylone}

Toujours selon Hérodote (I, 77), il s'agit de la dernière alliance conclue par Crésus : «[...] $\mu \varepsilon \tau \alpha-$

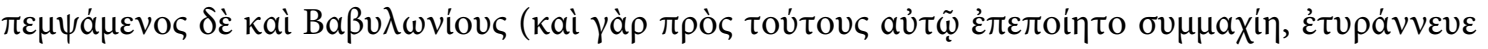

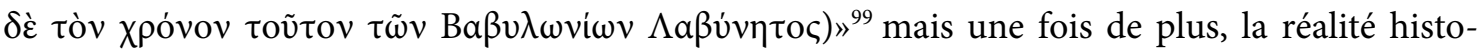
rique de tels accords pose question.

En effet, les seules deux sources mentionnant cette alliance sont Hérodote et Xénophon, ce dernier étant repris par Justin. Le récit de ce dernier est à rapprocher de l'Athénien qui prétendait déjà que Crésus avait secouru Babylone, victime d'une attaque de Cyrus : vaincu, le Lydien aurait regagné Sardes ${ }^{100}$.

${ }^{97}$ Lafont 2001, 50-53 ; Charpin 2019, 203-234.

${ }^{98}$ Les fouilles archéologiques n'ayant révélé, à ce jour, aucune trace de contacts diplomatiques entre la Lydie et l'Égypte. Voir le premier chapitre de Charpin 2019, 21-45. Par ailleurs, Hérodote ne mentionne pas cette alliance lorsqu'il relate règne d'Amasis dans son Enquête. Hérodote, II, 162-182.

99 «[...] de faire venir aussi les Babyloniens (car il avait conclu une alliance avec eux aussi, à ces temps là, celui qui régnait sur les Babyloniens était Labynète)». Sur ce Labynète, voire la note 3 . Le Labynète mentionné ici (I, 77) ne peut être que Nabonide, le roi de Babylone contemporain de la fin du règne de Crésus. Néanmoins, Hérodote mentionne deux autres Labynète : un premier qui aurait servi de médiateur lors de la conclusion de la paix entre les Mèdes et les Lydiens d'Astyage et d'Alyatte (I, 74) et un dernier Labynète, fils de Nitocris qui portait le nom de son père (I, 188). Selon Paul-Alain Beaulieu, l'hypothèse la plus probable serait que les deux premiers Labynète (I, 74 et I, 77) seraient la même personne : Nabonide ; pour le troisième $(\mathrm{I}, 188)$ il s'agirait de son fils Bel-šar-ușur. La confusion entre Nabonide et son fils Belšar-ușur est récurrente dans les sources grecques et hébraïques. Beaulieu 1989, 80-81. Cependant, concernant le Labynète de I, 74, le roi de Babylone à cette époque était Nabuchodonosor ; ainsi certains historiens estiment que derrière ce Labynète mentionné ici il faudrait reconnaître soit Nabuchodonosor, soit Nabonide qui aurait agi en tant que le représentant du roi de Babylone. Legrand 1932a, 77, note 4 et 183, note 1 ; Wiseman 1985, 8-9; Asheri et al. 2007, 135.

${ }^{100}$ Cette bataille de Babylone et ses préparatifs sont détaillés dans les six premiers livres de la Cyropédie. 
Justin, dans son abrégé des Histoires philippiques de Trogue Pompée qui résume les propos de Xénophon, nous transmet que :

Domitis deinde plerisque, cum aduersus Babylonios bellum gereret, Babyloniis rex Lydorum Croesus, cuius opes diuitiaeque insignes ea tempestate erant, in auxilium uenit; uictusque iam de se sollicitus in regnum refugit. Cyrus quoque post uictoriam, conpositis in Babylonia rebus, bellum transfert in Lydiam $^{101}$.

Dans ce récit, Justin ne mentionne pas le nom du souverain de Babylone. De même, il dit rien sur la raison qui pousse Crésus à venir en aide aux Babyloniens. Néanmoins, si le roi de Lydie décide de porter secours aux Chaldéens, c'est en vertu d'accords antérieurs. Or, il s'avère que cette campagne du roi perse mentionnée par Xénophon et par la source résumée par Justin à l'encontre du roi de Babylone est imaginaire. En effet, excepté Xénophon, aucune source antique (y compris la Chronique de Nabonide) ne mentionne une première campagne de Cyrus contre Babylone avant sa capture ${ }^{102}$.

Pour sa part, V. La Bua explique que l'alliance entre Crésus et Nabonide aurait été conclue pour régler la répartition des territoires mèdes, après la chute d'Astyage ca. 550 av. J.-C. Nabonide aurait soutenu Cyrus lors de sa guerre contre le roi des Mèdes car il voulait, lui aussi, rompre l'équilibre de la paix de 585 qui fixait la frontière entre la Lydie et la Médie sur le fleuve Halys et donc tirer profit de la chute d'Astyage pour agrandir son territoire, notamment afin de contrôler la région de Harrān ${ }^{103}$. Cependant, Nabonide, sans nouer d'alliance avec Cyrus, n'a rien entrepris pour aider Astyage ou même contrecarrer le roi perse ${ }^{104}$; car il n'est mentionné dans aucune source que Nabonide fournit une aide quelconque à Cyrus lors de sa marche sur Ecbatane. V. La Bua ne s'appuie donc sur aucun témoin pour asseoir son affirmation.

Maintenant que nous venons de constater que seul le récit d'Hérodote pouvait être vraiment pris en compte puisque ceux de Xénophon et Justin ont toute chance de n'être que des inventions, regardons de plus près les différentes relations diplomatiques entretenues et établies par Nabonide de Babylone au cours de son règne afin d'analyser avec plus de références par la suite la crédibilité des propos du Père de l'Histoire.

\section{Les relations diplomatiques établies par Nabonide de Babylone:}

Pour ce faire, tournons-nous vers les sources babyloniennes. Les principales informations disponibles pour les années comprises entre le début du règne de Nabonide en 556 av. J.-C. et la prise de Babylone par Cyrus en 539 av. J.-C. sont à trouver dans la Chronique de Nabonide, les Stèles

101 «Ensuite, comme, après la soumission de la plupart d'entre elles, Cyrus faisait la guerre contre les Babyloniens, le roi des Lydiens Crésus, dont les ressources et les richesses étaient immenses en ce tempslà, vint au secours des Babyloniens ; et vaincu, inquiet désormais pour lui-même, il s'enfuit dans son royaume. Quant à Cyrus, une fois mises en ordre les affaires en Babylonie après sa victoire, il transfère la guerre en Lydie». Justin, I, 7, 3-4 (éd. Arnaud-Lindet 2003).

${ }^{102}$ Anderson 1970, 170-171 ; Bizos 1971, VIII ; Beaulieu 1989, 197-200. Hérodote ne mentionne jamais une campagne de Cyrus contre Babylone avant sa marche contre Sardes. Briant 1996, 42-55.

${ }^{103}$ La Bua 1977, 34-37.

${ }^{104}$ Beaulieu 1989, 197-200 ; Briant 1996, 42. Sur l'alliance entre Crésus et Nabonide, voir aussi Högemann - Oettinger 2008, 395. 
de Harrā $n^{105}$, le Cylindre de Sippar ainsi que le Cylindre d'Ur. Pour la période qui nous intéresse ici, c'est-à-dire les années précédant la chute de Sardes (fixée en 547 av. J.-C.) ${ }^{106}$, et pour les années dont les lignes ne sont pas endommagées, il s'avère que la Chronique de Nabonide ${ }^{107}$ ne mentionne aucune alliance avec un souverain étranger. Pourtant, nous savons que par le passé les scribes babyloniens consignaient parfois les différentes alliances contractées par leurs souverains avec leurs homologues étrangers. En témoigne notamment la mention du traité d'amitié et de paix (țūbtu u sulummû) conclu en 614-613 av. J.-C. entre le roi des Mèdes Kyaxare et Nabopolassar le roi de Babylone, lors de leur campagne commune contre la cité de Ninive ${ }^{108}$. On est donc tenté de penser que si Nabonide avait conclu une alliance avec Crésus (ou même Cyrus), celle-ci aurait bien pu être consignée dans cette Chronique ${ }^{109}$, même si elle fut probablement réécrite après la chute de Babylone sous les coups des armées de Cyrus ${ }^{110}$.

En regard, un extrait des Stèles de Harrān datant du règne de Nabonide s'avère des plus instructifs. Nous reproduisons ici les deux traductions des deux textes conservés de ces Stèles de Harrān:

(...) Selon la parole de Sîn et d'Ishtar, la Maîtresse du combat, sans laquelle la paix et la guerre n'existent pas sur la terre, et sans laquelle aucune arme n'est forgée, elle étendit sa main sur eux, de telle sorte que les rois du pays d'Égypte, du pays des Mèdes, du pays des Arabes, et tous les rois qui étaient hostiles, pour la paix et d'amitié (sulummû u țūbāti), ont envoyé [des messagers] devant moi $(\ldots)^{111}$.

Ce texte lève donc un voile sur les relations diplomatiques au cours du règne de Nabonide, et plus précisément, à la lecture de l'intégralité de l'inscription, sur celles intervenues durant le long séjour du roi babylonien dans l'oasis de Tayma en Arabie (ca. 553-543 av. J.-C.) ${ }^{112}$. Au cours de cette tranche chronologique où Nabonide séjourne en Arabie, le roi d'Égypte n'est autre

${ }^{105}$ Pour la Chronique de Nabonide (ABC 7), nous avons utilisé l'édition ainsi que la traduction de Glassner 2004. Pour les inscriptions de Harrān, nous avons exploité l'édition et la traduction de Schaudig 2001.

${ }^{106}$ Date fixée grâce à la Chronique de Nabonide. Les dernières lectures et analyses de la Chronique de Nabonide tendent à prouver que durant la neuvième année du règne du roi babylonien (soit 547 av. J.-C.), Cyrus mena bel et bien campagne contre la Lydie. Voir Zawadzki 2010, 142-154; Van der Spek 2014, 256 ; Leloux 2018, p. 350-355 ; Adalı et al. 2017, 153-185.

${ }^{107} \mathrm{Du}$ fait du mauvais état de conservation de la stèle, les années 552 à 550 av. J.-C. ainsi que les années 544 à 539 av. J.-C. sont perdues.

${ }^{108}$ Chronique de la chute de Ninive (ABC 3), 1. 29. Texte édité et traduit par Grayson 1975, 93 et Glassner 1993, n. 22 - 2004, 218-225. On trouve aussi la mention de tels accords dans la Chronique d'Histoire synchronique (ABC 21), col. ii, 1. 1 ; col. iii, 1. 18.

${ }^{109}$ Il reste néanmoins les années perdues qui empêchent de rejeter totalement l'hypothèse.

${ }^{110}$ Waerzeggers 2015, 102-106.

${ }^{111}$ Schaudig 2001 txt. 3.1, col. i, 1l. 38-44. Traduction française basée sur la traduction allemande de H. Schaudig : "Auf das Wort Sîns und Ištars, der Herrin der Schlacht, ohne die Feindschaft und Friedensschluß im Lande nicht entstehen und die Waffen nicht geführt werden - sie streckte ihre Hand über sie aus, damit die Könige vom Lande Ägypten, vom L[and der Med]er, vom Land der Ar[aber und] die Gesamtheit der fei[nd]lichen Könige z[u] Friedens[schluß und guten Beziehungen (Boten)] vor mi[ch hinschickten]» : Schaudig 2001, 497.

${ }^{112}$ Gadd 1958, 75-78 ; Beaulieu 1989, 169-185 ; Zawadzki 2010, 20-26 ; Hausleiter 2012, 819-824. 
qu'Amasis (ca. 570-526 av. J.-C.), le seul pharaon contemporain de Nabonide. Cette inscription provenant de Harrān nous rapporte donc un envoi d'ambassadeurs de la part d'Amasis au roi de Babylone se trouvant à Tayma afin de conclure des traités «d'amitié et paix». Quant au roi de Médie mentionné dans l'inscription, il devrait s'agir en fait d'un des nombreux potentats qui se trouvaient auparavant sous la domination d'Astyage. On aurait ainsi un indice supplémentaire de l'organisation de cette confédération mède composée de roitelets devant prêter allégeance au roi des Mèdes ${ }^{113}$.

Sur ces indications, nous pouvons affirmer que cet envoi d'ambassadeurs mèdes auprès de Nabonide s'est déroulé entre 550 et 543 av. J.-C. (avant le retour du roi babylonien dans sa capitale), et même plus précisément dans les années 546-543 av. J.-C., ce qui nous situe après la chute de Sardes et de Crésus (datée de 547 av. J.-C.) ${ }^{114}$. En effet, la raison du traité «d'amitié et de paix» entre Nabonide et le potentat mède aurait eu plus justement pour objet l'occupation par les Babyloniens de Harrān (où se trouvait le temple du dieu Sîn) après la chute d'Astyage : depuis la chute de Ninive, cette région était sous contrôle mède ${ }^{115}$. Le temple de Sîn (dont le culte du dieu-lune était le favori du souverain de Babylone ainsi que de sa mère) avait été détruit lorsqu'il était passé sous contrôle mède et Nabonide y avait entrepris une grande opération de restauration $^{116}$. Ainsi, grâce à cet accord avec le «roi de Médie», Nabonide aurait eu le champ libre pour toute entreprise de restauration ainsi que l'opportunité de contrôler la zone de Harrān ${ }^{117}$. Sans doute ce traité a-t-il aussi été conclu afin de remettre en vigueur l'ancienne alliance babylomède passée en 613 av. J.-C. ${ }^{118}$.

Ajoutons que l'accord de «d'amitié et de paix» avec l'Égypte est à replacer dans le contexte des campagnes de Nabonide à l'ouest, jusqu'aux frontières égyptiennes ${ }^{119}$. Dès son accession au trône ca. 556 av. J.-C., le roi de Babylone avait entrepris des expéditions en Syrie (Amurri), en Cilicie $(H u-m e-e)$ et en Édom $(A-d u-u m)^{120}$. Ces campagnes devaient probablement inquiéter les Égyptiens qui contrôlaient encore une partie de la région jusque Gaza ${ }^{121}$. Ainsi, ils pouvaient encore craindre une attaque des armées babyloniennes sur leur territoire comme ce fut le cas sous le prédécesseur d'Amasis, Apriès. Par conséquent, Nabonide n'aurait pas pu rentrer à Ba-

113 Tuplin 1994, 255 ; Briant 1996, 912 ; Jursa 2003, 169 ; Waters 2011, 245-250 ; Leloux 2018, 304. Gadd pensait qu'il s'agissait en réalité de Cyrus qui aurait pris ce titre assez impersonnel après la chute d'Ecbatane. Gadd 1958, 76-77. Cette théorie est aujourd'hui largement rejetée.

${ }^{114}$ Les autres monarques qui auraient envoyé des ambassadeurs seraient des souverains contre lesquels Nabonide avait sans doute mené des expéditions lors de son séjour à Tayma. Wiseman 1991, 246- 248 ; Ruzicka 2012, 11-13.

${ }^{115}$ Cuyler Young 1988, 30-32 ; Tuplin 2004, 234.

${ }^{116}$ Schaudig 2001 txt. 3.1, col. i, 1l. 1-9 - Schaudig 2001, 487-488 et 496.

${ }^{117}$ Cuyler Young 1988, 30.

${ }^{118}$ Mais comme Nabonide n'a envoyé aucun renfort durant les conflits opposant Cyrus à Astyage entre 553 et 550 av. J.-C., on peut penser que cette alliance de 613 av. J.-C. n'était en réalité qu'une sorte d'accord de non-agression (échange de politesses).

${ }^{119}$ Cfr. supra.

${ }^{120}$ Chronique de Nabonide (ABC 7), col. i, 1l. 7-17.

${ }^{121}$ Hérodote III, 5. 
bylone sans s'être assuré que l'Égypte ainsi que les peuplades arabes ne tenteraient aucune action belliqueuse de ce côté ${ }^{122}$.

Concernant la nature de ces accords, nous pouvons au mieux émettre l'hypothèse qu'il ne s'agissait d'accords d'amitié et de non-agression (comprenant probablement de clauses «commerciales» ${ }^{123}$ que chacune des parties contractantes s'engageait à respecter. Cette conclusion d'alliance (qui consistait vraisemblablement en une prestation de serment) a dû être conclue à distance et être réalisée selon les usages en vigueur au Proche-Orient : ces serments (sans doute mis par écrit - probablement en akkadien - sur des tablettes d'argile ou de métal) devaient comporter une garantie divine ainsi que différentes clauses militaires, politiques, juridiques et commerciales $^{124}$. L'alliance conclue entre Amasis et Nabonide devait, en réalité, ne comporter que des clauses politiques (et commerciales, comme l'immunité conférée aux marchands). Les deux souverains se juraient ainsi de ne pas devenir hostile l'un envers l'autre, sans obligation de se porter au secours de l'autre partie en cas de conflit. Le fait qu'Amasis n'ait apporté aucune aide à Nabonide alors que Cyrus s'apprêtait à attaquer Babylone appuierait dès lors notre hypothèse. Amasis n'était pas précisément obligé de fournir une assistance militaire à un royaume envers lequel il s'était «simplement» engagé à reconnaître le roi (comme un «frère», un alter ego) et à ne pas intenter d'action militaire contre lui ${ }^{125}$. Cet accord devait ainsi permettre aux deux royaumes, qui voyaient la menace perse de plus en plus prégnante, de ne pas à devoir combattre sur plusieurs fronts à la fois s'ils étaient attaqués par les troupes de Cyrus.

\section{Avec la Lydie :}

Pour en revenir une nouvelle fois à Crésus, il existe deux hypothèses pour expliquer un éventuel accord conclu avec Nabonide.

Si l'alliance a été nouée entre l'accession au trône de Nabonide en 556 et la prise d'Ecbatane ca. 550 av. J.-C., sans doute était-ce un moyen pour Crésus d'établir une paix durable avec le souverain de Babylone. Peu avant la montée sur le trône de Nabonide, sous le commandement de Neriglissar ${ }^{126}$, les armées babyloniennes avaient mené des campagnes en Cilicie, région qui partageait une frontière commune avec les domaines sous contrôle du Mermnade ${ }^{127}$. Dès lors, Crésus aurait bien pu chercher à conclure une alliance qui aurait comporté des clauses politiques et probablement commerciales. Les deux souverains se seraient ainsi engagés à ne pas devenir hos-

${ }^{122}$ Ruzicka 2012, 12. De même, Amasis a pu envoyer une ambassade afin de s'assurer que Nabonide n'entreprendrait aucune attaque.

${ }^{123}$ Car on sait que l'Égypte qui exportait de grandes quantités d'alun et de lin en Babylone entretenait de solides liens commerciaux également avec la Syrie, Phénicie, Élam, Chypre, et l'Asie Mineure. Wiseman 1991, 272-275. Ainsi Amasis voulait-il encore une fois s'assurer de la viabilité des bonnes affaires de ses commerçants ainsi que de la prospérité de Naucratis.

${ }^{124}$ Sur la forme et contenu des traités au Proche-Orient, voir Charpin 2019, 127-168. Sur les garanties divines, voir Charpin 2019, 169-202.

${ }^{125}$ Il faut noter l'emploi des mêmes termes «țūbtu » et («țūbāti ») «sulummûu » pour désigner l'alliance conclue en 613 av. J.-C. par Nabopolassar et Kyaxare et celle mentionnée sur les Stèles de Harrān. Ce qui nous conforte dans l'idée qu'il s'agit du même type d'alliance à savoir de «non-agression» mutuelle.

${ }^{126}$ La Chronique des trois premières années de Neriglissar (ABC 6), 1l. 1-27. Voir Glassner 2004, 232-233.

${ }^{127}$ Hérodote, I, 28. 
tiles l'un envers l'autre. Par la même occasion, le roi de Lydie s'assurait la libre circulation des produits échangés tant par voie maritime (grâce au commerce des cités grecques sous domination mermnade) que par voie terrestre. Cette alliance rappelle celle conclue entre la Lydie et la Médie en 585 av. J.-C. ${ }^{128}$ en se liant avec Nabonide au moyen de ce type d'entente de "non-agression», Crésus se serait assuré des ouvertures commerciales en Médie et en Babylonie. L'absence d'aide envoyée par Nabonide à Crésus, ainsi que l'absence d'action des armées babyloniennes lorsque Cyrus traverse le territoire babylonien pour se rendre en Lydie ${ }^{129}$, appuierait l'idée que cet accord ne comportait aucune clause militaire voulant que les deux contractants se portent assistance en cas de conflit. Cependant, une fois de plus, rien ne permet de prouver la réalité historique d'un tel accord.

D'autre part, si l'éventuelle alliance a été conclue après la chute d'Astyage sous les coups des armées perses de Cyrus ca. 550 av. J.-C., peut-être était-ce également un accord de «non-agression réciproque». Crésus se serait ainsi assuré de ne pas subir d'attaque de la part des armées babyloniennes pendant sa campagne en Ptérie et en Cappadoce. Ici aussi, l'accord n'aurait comporté que des clauses politiques (non-agression mutuelle) et commerciales.

Dans ce cas-ci également, dans l'état actuel des sources à notre disposition, il n'existe aucune trace de tels accords, si jamais ils furent réellement noués entre les deux parties. Seul Hérodote mentionne cette alliance.

\section{Conclusions}

Comme nous venons de le proposer, les seuls liens attestés entre Crésus, Amasis et Nabonide sont ceux qui ont lié le pharaon au roi de Babylone. Même si nous ne connaissons rien de la teneur de tels accords, il se pourrait que ceux-ci n'aient été que des conventions très générales d'amitié et de non-agression réciproques où les rois se reconnaissaient comme «frères» dans le but vraisemblablement d'assurer la sécurité de leurs possessions ainsi que le bon accueil de leurs commerçants.

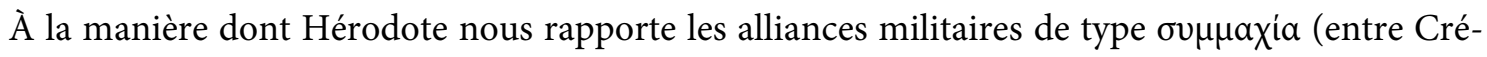
sus, Babylone et l'Égypte), il nous est impossible d'affirmer leur réalité historique. Nous pouvons même aller jusqu'à mettre l'existence de ce type de traités d'alliance militaire en doute, dans la mesure où aucun des «alliés» ne vint prêter main forte à Crésus lorsqu’il fut assiégé dans sa citadelle de Sardes.

En revanche, comme nous venons de le voir, il est permis d'envisager que Crésus ait bien pu conclure une alliance avec Amasis. Celle-ci, de nature défensive, devait permettre aux deux parties de se prémunir en cas d'attaque d'un un ennemi commun : le royaume de Babylone. Mais les accords de non-agression réciproque noués ultérieurement par Crésus avec Nabonide de Babylone auraient ainsi rendu caduque l'alliance avec Amasis. Raison pour laquelle le pharaon n'aurait envoyé aucune aide lors de la campagne du roi lydien en Ptérie et lors du siège de Sardes par les troupes perses.

\footnotetext{
${ }^{128}$ Hérodote, I, 74. Accord étudié par Leloux 2016 ; Leloux 2017.

${ }^{129}$ Chronique de Nabonide (ABC 7), col. ii, 1l. 15-16.
} 
Par ailleurs, nous savons qu'Hérodote avait rédigé des logoi sur la Lydie, l'Égypte et la Babylonie pour exalter la grandeur et la puissance de ces vieux royaumes ${ }^{130}$. La nouvelle de la chute de Crésus en Lydie (qui passait pour le plus riche) et celle d'Amasis en Égypte, personnages qui avaient l'un et l'autre fait des cadeaux mémorables aux Grecs, a rudement frappé l'imagination de ces derniers pour qui il était difficile d'envisager une puissance supérieure aux Lydiens et aux Égyptiens. C'est justement parce que les Grecs ont vite pris pleine conscience de ce que signifiaient les chutes de Sardes, de Babylone et de l'Égypte que leurs victoires ultérieures sur les Perses ressortent comme autant d'événements sans parallèle depuis la guerre de Troie ${ }^{131}$.

En conclusion, il est de notre avis qu'Hérodote pourrait avoir forcé le trait et transformé de simples convenances diplomatiques entre Crésus, Babylone et l'Égypte en des alliances militaires de type $\sigma v \mu \mu \alpha \chi_{i} \alpha$ afin d'expliciter plus ouvertement à son public l'existence de liens entre les trois plus grandes puissances de l'époque. Ces trois grandes puissances n'avaient pas réussi à s'opposer à Cyrus le Grand et aux Perses contrairement aux Grecs qui y étaient parvenus.

Faut-il le dire, tous ces propos conféraient plus d'éclat encore aux victoires de Marathon, de Salamine et de Platées qu'Hérodote exposait dans la seconde partie de son Enquête ${ }^{132}$. Mais pardessus tout, le tableau ainsi dressé par l'historien immortalisait la suprématie des Grecs sur les plus puissants des Barbares.

\section{Bibliographie}

Adalı et al. 2017

S. F. Adalı - M. F. Demirci - A. M. Özbayoğlu, Automated Image Matching and New Readings for Cyrus the Great's 547 BC Campaign in the Nabonidus Chronicle $($ BM $35382=$ ABC 7), Die Welt des Orients 47-2, 2017, 153-185.

Adcock - Mosley 1974

F. Adcock - D. J. Mosley, Diplomacy in Ancient Greece, London 1974.

Agut-Labordère 2012a $\quad$ D. Agut-Labordère, Plus que des mercenaires! L'intégration des hommes de guerre grecs au service de la monarchie saïte, Pallas 89 , 2012, 293-306.

Agut-Labordère 2012b

D. Agut-Labordère, Le statut égyptien de Naucratis, in : V. Dieudonné et al. (éd.), Entités locales et pouvoir central: La cité dominée dans l'Orient hellénistique, Nancy 2012, 353-373.

${ }^{130}$ Drews 1973, 66-67. Néanmoins, pour Félix Jacoby, Hérodote avait rédigé ses logoi barbares à la suite de ses voyages; son but premier était de faire œuvre ethnographique à la façon d'Hécatée de Milet. Ce n'est qu'à son arrivée à Athènes qu'Hérodote se mit à traiter des guerres médiques. Jacoby 1913.

${ }^{131}$ Drews 1973, 66-67 ; Fantalkin 2014, 32. Selon A. Fantalkin, les arguments de Robert Drews vont de pair avec ceux de Jonathan Hall qui déclare que ce sont ces confrontations entre les Hellènes et les Perses lors des guerres médiques qui ont été l'événement principal de la création de l’identité grecque. Hall 2002.

${ }^{132}$ Cette victoire grecque sur les Perses a été l'événement cristallisateur de la conscience «nationale» grecque ; Elle a été la raison pour laquelle Hérodote a voulu mettre en avant les megala erga de ces royaumes barbares qui furent défaits par les Perses, afin de rendre encore plus uniques ces victoires des Grecs sur les armées de Darius et Xerxès. Fantalkin 2014, 32. 
Anderson 1970

Anderson 2008

Arnaud-Lindet 2003

Asheri et al. 2007

Azoulay 2004

Balandier 2009

Baurain 1997

Beaulieu 1989

Bederman 2001

Bizos 1971

Bolshakov 2010

Braun 1982

Bresson 1980

Bresson 2005

Briant 1996

Canfora 1994
J. K. Anderson, Military Theory and Practice in The Age of Xenophon, Berkeley-Los Angeles 1970.

J. K. Anderson, Xenophon (1 $1^{\text {ère }}$ éd. 1974), London $2008^{2}$.

M. P. Arnaud-Lindet, Marcus Junianus Justinus. Abrégé des Histoires Philippiques de Trogue Pompée, texte établi et traduit par M. P. Arnaud-Lindet, in: Corpus Scriptorum Latinorum, A digital library of Latin litterature, [en ligne], http://www.forumromanum. org/literature /justin/trad1.html.

D. Asheri - A. Lloyd - A. Corcella, A commentary on Herodotus. Books I-IV, édité par O. Murray, A. Moreno, avec la contribution de M. Brosius, Oxford 2007.

V. Azoulay, Xénophon et les grâces du pouvoir. De la charis au charisme, Paris 2004.

C. Ballandier, L'Égypte, Chypre et la route de Péluse à Gaza: approche micro-régionale des politiques stratégiques des derniers souverains égyptiens et achéménides (610-332 av. J.-C.), in: D. Michaelides et al. (ed.), Egypt and Cyprus in Antiquiy, Oxford 2009, 78-96.

C. Baurain, Les Grecs et la Méditerranée orientale. Des siècles obscurs à la fin de la période archaïque, Paris 1997.

P. A. Beaulieu, The Reign of Nabonidus. King of Babylon 556-539

B.C., New Haven-London 1989.

D. J. Bederman, International law in Antiquity, Cambridge 2001.

M. Bizos, Xénophon. Cyropédie, I, Livres I et II, texte établi et traduit par M. Bizos, Paris 1971.

A. O. Bolshakov, Persians And Egyptians: Cooperation In Vandalism?, in: S. H. D’Auria (ed.), Offerings To The Discerning Eye. An Egyptological Medley in Honor of Jack A. Josephson, LeidenBoston 2010, 45-53.

T. F. R. G. Braun, The Greeks in Egypt, in: J. Boardman - N. G. L. Hammond (éd.), The Cambridge Ancient History, III-3, The Expansion of the Greek World, Eight to Sixth Centuries B.C., Cambridge 1982, 32-56.

A. Bresson, Rhodes, l'Héllénion et le statut de Naucratis (VIe-IVe siècles a.C.), DHA 6, 1980, 291-349.

A. Bresson, Naucratis: de l'emporion à la cité, Topoi 12/13-1, 2005, 133-155.

P. Briant, Histoire de l'Empire perse. De Cyrus à Alexandre, Paris 1996.

L. Canfora, Histoire de la littérature grecque d'Homère à Aristote, traduit de l'italien par D. Fourgous, Paris 1994. 
Charpin 2019

Colin 1998

Cook 1982

Couvenhes 2016

Crahay 1956

Cuyler Young 1988

Daux 1957

Delebecque 1957

De Meulenaere 1975

Dillery 2005

Drews 1973

Due 1989

Elayi 2018

Fantalkin 2014

Fehling 1989

Francis - Vickers 1984
D. Charpin, «Tu es de mon sang». Les alliances dans le ProcheOrient ancien, Paris 2019.

F. Colin, Les fondateurs du sanctuaire d'Amon à Siwa (Désert Libyque) Autour d'un bronze de donation inédit, in: E. Clarisse et alii (ed.), Egyptian Religion. The Last Thousand Years. Tome I, Studies dedicated to the Memory of Jan Quaegebeur, Leuven 1998, 329-354.

J. M. Cook, The Easterns Greeks, in: J. Boardman - N. G. L. Hammond (éd.), The Cambridge Ancient History, III-3, The Expansion of the Greek World, Eight to Sixth Centuries B.C., Cambridge 1982, 196-221.

J. C. Couvenhes, La symmachia comme pratique du droit international dans le monde grec, Dialogues d'histoire ancienne supplément 16, 2016, 13-49.

R. Crahay, La littérature oraculaire chez Hérodote, Paris 1956.

T. Cuyler Young, The early history of the Medes and the Persians and the Achaemenid empire to the death of Cambyses, in: J. Boardman - N. G. L. Hammond - D. M. Lewis - M. Ostwals (éd.), The Cambridge Ancient History, IV, Persia, Greece and Western Mediterranea c. 525 to 479 B.C., Cambridge 1988, 1-52.

G. Daux, Remarques sur la composition du Conseil amphictionique, Bulletin de correspondance hellénique 81, 1957, 95-120.

E. Delebecque, Essai sur la vie de Xénophon, Paris 1957.

H. De Meulenaere, Amasis, in: W. Helck (éd.), Lexikon der Ägyptologie, I, A-Ernste, Wiesbaden 1975, col. 182.

J. Dillery, Greek Sacred History, The American Journal of Philology 126-4, 2005, 505-526.

R. Drews, The Greek Accounts of Eastern History, Cambridge (Ma) 1973.

B. Due, The Cyropaedia. Xenophon's aims and methods, Aarhus 1989.

J. Elayi, Histoire de la Phénicie, Paris 2018.

A. Fantalkin, Naukratis as a contact zone: Revealing the Lydian connection, in: R. Rollinger - K. Schnegg (ed.), Kulturkontakte in Antiken Welten, vom Denkmodell zum Fallbeispeil, Proceedings des internationalen Kolloqiums aus Anlass des 60. Gebuststages von Christoph Ulf, Innsbruck, 26. Bis 30, Januar 2009, LeuvenParis-Walpole 2014, 17-52.

D. Fehling, Herodotus and his «Sources»: citation, invention and narrative art, traduit de l'allemand par J. G. Howie, Leeds 1989.

E. D. Francis - M. Vickers, Green Goddess: A Gift to Lindos from Amasis of Egypt, American Journal of Archaeology 88-1, 1984, 6869. 
Gadd 1958

Germain 1954

Giovannini 2007

Glassner 1993

Glassner 2004

Grayson 1975

Greaves 2002

Greaves 2010

Hall 2002

Hausleiter 2012

Hawkins 1982

Herman 1987

Higbie 2003

Högemann - Oettinger

2018

Intrieri 2010

Intrieri 2013
C. J. Gadd, The Harran Inscriptions of Nabonidus, Anatolian Studies 8, 1958, 35-92.

G. Germain, La mystique des nombres dans l'épopée homérique et sa préhistoire, Paris 1954.

A. Giovannini, Les relations internationales entre États dans la Grèce antique du temps d'Homère à l'intervention romaine (ca. 700-200 av. J.-C.), Stuttgart 2007.

J. J. Glassner, Chroniques mésopotamiennes, Paris 1993.

J. J. Glassner, Mesopotamian Chronicles, edited by B. R. Foster, Atlanta 2004.

A. K. Grayson, Assyrian and Babylonian Chronicles, text, translation and commentary by A. K. Grayson, New York 1975.

A. M. Greaves, Miletos. A history, London \& New-York 2002.

A. M. Greaves, The land of Ionia. Society and Economy in the Archaic Period, Chichester-Malden 2010.

J. M. Hall, Hellenicity. Between Ethnicity and Culture, ChicagoLondon 2002.

A. Hausleiter, North Arabian Kingdom, in: D. T. Potts (éd.), A Companion to the Archaeology of the Ancient Near East, vol. 1, Malden-Oxford-Chichester 2012, 816-832.

J. D. Hawkins, The Neo-Hittite states in Syria and Anatolia, in: J. Boardman - I. E. S. Edwards - N. G. L. Hammond - E. Sollberger (éd.), The Cambridge Ancient History, III-1, The Prehistory of the Balkans; and the Middle East and the Aegean world, tenth to eighth centuries B.C, Cambridge 1982, 372-471.

G. Herman, Ritualised Friendship and the Greek City, Cambridge 1987.

C. Higbie, The Lindian Chronicle and the Greek Creation of their Past, Oxford 2003.

P. Högemann - N. Oettinger, Lydien. Ein altanatolischer Staat zwischen Griechenland und dem Vorderen Orient, Berlin/Boston 2018.

M. Intrieri, Philoi kai xeinoi.sepisui rapporti fra tiranni e basileis in Erodoto, in : M. Caccamo Caltabiano, C. Raccuia, E. Santagati (éd.), Tyrannis, Basileia, Imperium: forme, prassi e simboli del potere politico nel mondo greco e romano. Atti delle Giornate seminariali in onore di S. Nerina Consolo Langher, Messina, 17-19 dicembre 2007. Pelorias, 18. Messina 2010, 123-142.

M. Intrieri, Intessere relazioni. Osservazioni sull'itinerario di philia (I. dalle origini al V sec. a.C.), in Historiká III, 2013, 213-271. 
Jacoby 1913

James 1991

Jansen-Winkeln 2014

Joannès 1991

Joannès 2008

Jursa 2003

Kaplan 2016

La Bua 1977

Lafont 2001

Legrand 1932a

Legrand 1932b

Leloux 2014

Leloux 2016

Leloux 2017
F. Jacoby, Herodotos, in: Pauly Realencyclopadie der classischen Altertumswissenschaft, Supplementband II, Herodes bis Herodotos, Stuttgart 1913, col. 205-520.

T. G. H. James, Egypt: The Twenty-fifth and Twenty-sixth dynasties, in: J. Boardman - I. E. S. Edwards - N. G. L. Hammond - E. Sollberger - C. B. F. Walker (éd.), The Cambridge Ancient History, III-2, The Assyrian and Babylonian Empires and other States of The Near East, from the Eighth to the Sixth Centuries B.C., Cambridge 1991, 677-747.

K. Jansen-Winkeln, Die Siegesstele des Amasis, Zeitschrift für Ägyptische Sprache und Altertumskunde 141-2, 2014, 132-153.

F. Joannès, L'Asie Mineure méridionale d'après la documentation cunéiforme d'époque néo-babylonienne, Anatolia Antiqua. Eski Anadolu 1, 1991, 261-266.

F. Joannès, La stratégie des rois néo-babyloniens contre l'Assyrie, de 616 à 606 av. J.-C., in: P. Abrahami - L. Battini (éd.), Les armées du Proche-Orient ancien: $\mathrm{III}^{\mathrm{e}}-\mathrm{I}^{\mathrm{er}}$ mill. av. J.-C., Actes du Colloque International Organisé à Lyon les $1^{\text {er }}$ et 2 décembre 2006, Maison de l'Orient et de la Méditerranée 2008, 207-218 (Bar International Series 1855).

M. Jursa, Observations on the Problem of the Median 'Empire' on the Basis of Babylonian Chronicles, in: G. B. Lanfranchi et al. (éd.), Continuity of Empire: Assyria, Media, Persia, Padova 2003, 169179.

P. Kaplan, The Ring of Polycrates: friendship and alliance in the east Mediterranean, in Journal of Ancient History 4-2, 2016, 132157.

V. La Bua, Gli Ioni e il conflitto Lidio - Persiano, Miscellanea Greca e Romana V, 1977, 1-64.

B. Lafont, International relations in the ancient Near East: The birth of a complete diplomatic system, Diplomacy and Statecraft 12-1, 2001, 39-60.

P. E. Legrand, Hérodote. Histoires, I, Livre I : Clio, texte établi et traduit par P. E. Legrand, Paris 1932.

P. E. Legrand, Hérodote. Histoires, Introduction, texte établi par P. E. Legrand, Paris 1932.

K. Leloux, L'alliance lydo-spartiate, Ktèma 39, 2014, 271-288.

K. Leloux, The Battle of the Eclipse (May 28, 585 BC): A Discussion of the Lydo-Median Treaty and the Halys Border, Polemos 19-2, 2016, 31-54.

K. Leloux, L'Halys chez Hérodote, Studia Hercynia 21-1, 2017, 15-

24. 
Leloux 2018

Lenfant 2011

Malkin 1989

Massart 2006

Masson 1971

Novotny - Jeffers 2018

Redon 2012

Roux 1995

Ruzicka 2012

Sánchez 2001

Schaudig 2001

Schmitt-Pantel 1990

Sekunda 1985
K. Leloux, La Lydie d'Alyatte et Crésus. Un royaume à la croisée des cités grecques et des monarchies orientales. Recherches sur son organisation interne et sa politique extérieure, Thèse de Doctorat, inédit, Liège 2018.

D. Lenfant, Hérodote d'Halicarnasse, in: D. Lenfant (dir.), Les Perses vus par les Grecs. Lire les sources classiques sur l'Empire achéménide, Paris 2011, 214-227.

I. Malkin, Delphoi and the founding of social order in archaic Greece, Mètis 4-1, 1989, 129-153.

N. Massart, La «Chronique de Lindos»: un catalogue à la gloire du sanctuaire d'Athéna Lindia, Kernos 19, 2006, 229-243.

O. Masson, les Chypriotes en Égypte, Bulletin de la Société française d'égyptologie 60, 1971, 28-46.

J. Novotny - J. Jeffers, The Royal Inscriptions of Ashurbanipal (668-631 BC), Assur-etal-ilani (630-627 BC), and Sin-sarra-iskun (626-612 BC), Kings of Assyria, Volume 5/I, texte édité et traduit par J. Novotny et J. Jeffers, Winona Lake, 2018 (The Royal Inscriptions of the Neo-Assyrian Period. Les textes édités et traduits sont aussi disponibles en ligne sur The Royal Inscriptions of the NeoAssyrian Period, RINAP 5: The Royal Inscriptions of Ashurbanipal, Aššur-etel-ilāni, and Sîn-šarra-iškun, [en ligne], http://oracc.museum.upenn.edu/rinap/rinap5/. (Page consultée le 25 mai 2021).

B. Redon, L'identité grecque de Naucratis. Enquête sur la fabrication de la mémoire d'une cité grecque du Delta égyptien aux époques hellénistique et romaine, Revue des Études grecques 1251, 2012, 55-93.

G. Roux, La Mésopotamie, Paris 1995.

S. Ruzicka, Trouble in the West. Egypt and the Persian Empire 525-332 BCE, Oxford 2012.

P. Sanchez, L'amphictionie des Pyles et de Delphes. Recherches sur son rôle historique, des origines au $\mathrm{II}^{\mathrm{e}}$ siècle de notre ère, Stuttgart 2001.

H. Schaudig, Die Inschriften Nabonids von Babylon und Kyros' des Großen, samt den in ihren Umfeld entstandenen Tendenzschriften. Textausgabe und Grammatik, Münster 2001.

P. Schmitt-Pantel, [compte rendu sur] G. Herman, Ritualised Friendship and the Greek City, Cambridge, 1987, Annales. Économie, Sociétés, Civilisations 45-4, 1990, 878-879.

V. Sekunda, Achaemenid colonization in Lydia, Revue des Études Anciennes 87-2, 1985, 7-29. 
Sordi 1957

Spalinger 1977

Spalinger 1978

Tatum 1989

Tuplin 1994

Van der Spek 2014

Waerzeggers 2015

Waters 2011

Winand 2017

Wiseman 1985

Wiseman 1991

Zawadzki 2010
M. Sordi, La fondation du collège des naopes et le renouveau politique de l'Amphictionie au IV ${ }^{\mathrm{e}}$ siècle, Bulletin de correspondance hellénique 81, 1957, 38-75.

A. Spalinger, Egypt and Babylonia: A Survey (c. 620 B.C.-550 B.C.), Studien zur altägyptischen Kultur 5, 1977, 221-244.

A. Spalinger, The Date of the Death of Gyges and Its Historical Implications, Journal of the American Oriental Society 98-4, 1978, 400-409.

J. Tatum, Xenophon's Imperial Fiction. On the Education of Cyrus, Princeton 1989.

C. J. Tuplin, Persians as Medes, ArchHist 8, 1994, 235-256.

R. J. Van der Spek, Cyrus the Great, Exiles, and Foreign Gods: A Comparison of Assyrian and Persian Policies on Subject Nations, in: M. Kozul et al. (éd.), Extraction \& Control. Studies in Honor of Matthew W. Stolper, Chicago 2014, 233-264.

C. Waerseggers, Facts, Propaganda, or History? Shaping Political Memory in the Nabonidus Chronicle, in: J. M. Silverman - C. Waerzeggers (éd.), Political Memory in and after the Persian Empire, Atlanta 2015, 95-124.

M. Waters, Notes on the Medes and their "Empire" from JER 25:25 to HDT 1.134, in: G. Frame et alii (éd.), A Common Cultural Heritage: Studies on Mesopotamia and the Biblical World in Honor of Barry L. Eichler, Bethesda 2011, 243-253.

J. Winand, Une histoire personnelle des pharaons, Paris 2017.

D. J. Wiseman, Nebuchadrezzar and Babylon, Oxford 1985.

D. J. Wiseman, Babylonia 605-539 B.C., in: J. Boardman - I. E. S. Edwards - N. G. L. Hammond - E. Sollberger - C. B. F. Walker (éd.), The Cambridge Ancient History, III-2, The Assyrian and Babylonian Empires and other States of The Near East, from the Eighth to the Sixth Centuries B.C., Cambridge 1991, 229-251.

S. Zawadzki, The Portrait of Nabonidus and Cyrus in Their(?) Chronicle: When and Why the Present Version Was Composed, in: P. Charvát - P. Maříková Vlčková (ed.), Who Was King? Who Was Not King. The Rulers and their Ruled in the Ancient Near East, Prague 2010, 142-154. 


\section{Lydia-Mısır ve Lydia-Babil İttifakları \\ Özet}

Bu makale, Lydia kralı Kroisos’un Mısır kralı Amasis ile ve Babil kralı Nabonid ile yaptığı söylenen ve kabul etmek durumunda kaldığımız ittifakların güvenilirliğini araştırmaktadır. Aynı zamanda, Amasis'in Kyrene ve Samoslu Polykrates ile olan diplomatik ilişkilerinin yanı sıra Lydia Krallığı ve Mısır arasındaki bağlantı da incelenmiştir. Bu çalışma ayrıca Babil Kralı tarafından oluşturulan diplomatik bağlara da odaklanarak Kroisos ile olan bağlantılarının doğasını tespit etmeyi amaçlamaktadır.

Anahtar Sözcükler: Kroisos, Amasis, Nabonid, Lydia Krallığı, Müttefiklik.

\section{The Lydo-Egyptian and the Lydo-Babylonian Alliances}

\section{Abstract}

This article analyses the credibility given to the alliances, which were supposedly formed by the king of Lydia Croesus with Amasis of Egypt and with Nabonidus king of Babylon. At the same time, the diplomatic relations of Amasis with Cyrene and with Polycrates of Samos are studied, as well as the connection between the Lydian Kingdom and Egypt. Further, this study focuses also on the diplomatic ties built by the king of Babylon in order to determine what was the nature of the links between him and Croesus.

Keywords: Croesus, Amasis, Nabonidus, Lydian Kingdom, Alliance. 